\title{
The logical difference problem for description logic terminologies
}

\author{
Boris Konev, Dirk Walther, and Frank Wolter \\ University of Liverpool, Liverpool, UK \\ $\{$ konev, dwalther, wolter $@$ liverpool.ac.uk
}

\begin{abstract}
We consider the problem of computing the logical difference between distinct versions of description logic terminologies. For the lightweight description logic $\mathcal{E} \mathcal{L}$, we present a tractable algorithm which, given two terminologies and a signature, outputs a set of concepts, which can be regarded as the logical difference between the two terminologies. In particular, it decides whether they imply the same concept implications in the signature. A prototype implementation CEX of this algorithm is presented and experimental results based on distinct versions of SNOMED CT, the Systematized Nomenclature of Medicine, Clinical Terms, are discussed. Finally, results regarding the relation to uniform interpolants and possible extensions to more expressive description logics are presented.
\end{abstract}

\section{Introduction}

The standard diff operation for text files is an indispensable tool for comparing different versions of texts, and similar operations are available to software engineers comparing distinct versions of code produced in collaborative software projects. As observed, e.g., in [14], such a purely syntactic diff operation is hardly useful if the text consists of a set of axioms of an ontology. In this case, one is usually not interested in a comparison of the syntactic form of axioms, but in the consequences that the ontologies have. The authors of [14] present a number of heuristic rules to address this problem and develop a diff operator for ontologies. Except theoretical results in $[12,13,9]$, we are not aware of any logic-based approach to computing the logical diff of ontologies.

Our formalisation of the logical difference problem is based on the observation that when comparing distinct versions of ontologies one should take into account their signatures. In fact, the interesting differences between ontologies are those formulated in their shared signature (or even subsets thereof), and not those involving symbols used only in one of the two ontologies. Thus, the proposed notion of logical difference is based on the notion of $\Sigma$-entailment: an ontology $\mathcal{T} \Sigma$-entails an ontology $\mathcal{T}^{\prime}$ for a signature $\Sigma$, if for all concept implications $C \sqsubseteq D$ in $\Sigma, \mathcal{T}^{\prime}=C \sqsubseteq D$ implies $\mathcal{T} \models C \sqsubseteq D$. If $\mathcal{T}$ and $\mathcal{T}^{\prime}$ mutually $\Sigma$-entail each other, then they are called $\Sigma$-inseparable. By taking $\Sigma$ as the set of shared symbols of $\mathcal{T}$ and $\mathcal{T}^{\prime}, \Sigma$-inseparability means that $\mathcal{T}$ and $\mathcal{T}^{\prime}$ are 
not distinguishable by means of derivable concept implications in their shared signature. In this case, their logical difference will be regarded as empty.

We show that deciding $\Sigma$-entailment is tractable for $\mathcal{E} \mathcal{L}$-terminologies, i.e., sets of possibly cyclic concept definitions in the lightweight description logic $\mathcal{E} \mathcal{L}$; see $[1,10]$. Observe that for ontologies formulated as general TBoxes in description logics, the computational complexity of deciding $\Sigma$-entailment is by at least one exponential harder than the deduction problem, e.g., it is 2ExPTIMEcomplete for expressive description logics such as $\mathcal{A L C}, \mathcal{A L C Q}$, and $\mathcal{A L C} \mathcal{Q} \mathcal{I}[6$, 12] and ExpTIme-complete for $\mathcal{E} \mathcal{L}$ itself [13]. Moreover, even in such simple formalisms as acyclic propositional Horn Logic $\Sigma$-entailment is CO-NP-complete [5].

In applications, it is not enough to decide whether two ontologies are logically different, but an informative list of differences is required. We show that for any concept implication $C \sqsubseteq D$ in the logical difference between two $\mathcal{E} \mathcal{L}$ terminologies, there exist subconcepts $C^{\prime}$ and $D^{\prime}$ of $C$ and $D$, respectively, such that $C^{\prime} \sqsubseteq D^{\prime}$ is in the logical difference and $C^{\prime}$ or $D^{\prime}$ is a concept name. Thus, listing the set of all concept names involved in such implications appears to be an informative approximation of the logical difference between two $\mathcal{E} \mathcal{L}$-terminologies. This list is empty if, and only if, there is no logical difference between the two terminologies.

The system CEX implements, by employing a dynamic programming approach, the algorithm deciding $\Sigma$-entailment and lists the set of logical differences described above for acyclic $\mathcal{E} \mathcal{L}$-terminologies. We present a variety of experiments in which CEX is applied to different versions of SNOMED CT, the Systematized Nomenclature of Medicine, Clinical Terms. This terminology comprises $\sim 0.4$ million terms and underlies the systematized medical terminology used in the health systems of the US, the UK, and other countries [17].

Finally, we discuss an alternative approach to deciding $\Sigma$-entailment using uniform interpolants and explore the complexity of corresponding reasoning problems for acyclic $\mathcal{A L C}$-terminologies.

\section{Preliminaries}

Let $\mathrm{N}_{\mathrm{C}}$ and $\mathrm{N}_{\mathrm{R}}$ be countably infinite and disjoint sets of concept names and role names, respectively. In the description logic $\mathcal{E} \mathcal{L}$, concepts $C$ are built according to the syntax rule

$$
C::=\top|A| C \sqcap D \mid \exists r . C,
$$

where $A$ ranges over $\mathrm{N}_{\mathrm{C}}, r$ ranges over $\mathrm{N}_{\mathrm{R}}$, and $C, D$ range over concepts. The semantics of concepts is defined by means of interpretations $\mathcal{I}=\left(\Delta^{\mathcal{I}}, \mathcal{I}^{\mathcal{I}}\right)$, where the interpretation domain $\Delta^{\mathcal{I}}$ is a non-empty set, and ${ }^{\mathcal{I}}$ is a function mapping each concept name $A$ to a subset $A^{\mathcal{I}}$ of $\Delta^{\mathcal{I}}$ and each role name $r^{\mathcal{I}}$ to a binary relation $r^{\mathcal{I}} \subseteq \Delta^{\mathcal{I}} \times \Delta^{\mathcal{I}}$. The function ${ }^{\mathcal{I}}$ is inductively extended to arbitrary concepts by setting ${\top^{\mathcal{I}}}^{\mathcal{I}}:=\Delta^{\mathcal{I}},(C \sqcap D)^{\mathcal{I}}:=C^{\mathcal{I}} \cap D^{\mathcal{I}}$, and $(\exists r . C)^{\mathcal{I}}:=\left\{d \in \Delta^{\mathcal{I}} \mid\right.$ $\left.\exists e \in C^{\mathcal{I}}:(d, e) \in r^{\mathcal{I}}\right\}$.

A general TBox is a finite set of axioms, where an axiom can be either a concept inclusion (CI) $C \sqsubseteq D$ or a concept equality $(C E) C \equiv D$, where $C, D$ 
are concepts. An interpretation $\mathcal{I}$ satisfies a CI $C \sqsubseteq D$ (written $\mathcal{I} \models C \sqsubseteq D$ ) if $C^{\mathcal{I}} \subseteq D^{\mathcal{I}}$; it satisfies a CE $C \equiv D$ (written $\mathcal{I}=\bar{C} \equiv D$ ) if $C^{\mathcal{I}}=D^{\mathcal{I}}$. $\overline{\mathcal{I}}$ is a model of a general TBox $\mathcal{T}$ if it satisfies all axioms in $\mathcal{T}$. We write $\mathcal{T} \models C \sqsubseteq D$ $(\mathcal{T} \models C \equiv D)$ if every model of $\mathcal{T}$ satisfies $C \sqsubseteq D(C \equiv D$, respectively).

Our main concern in this paper are terminologies, i.e., general TBoxes $\mathcal{T}$ satisfying the following two conditions:

- $\mathcal{T}$ consists of CEs of the form $A \equiv C$ (concept definitions) and CIs of the form $A \sqsubseteq C$ (primitive concept definitions) only, where $A$ is a concepts name;

- no concept name occurs more than once on the left hand side of an axiom in $\mathcal{T}$.

Define the relation $\prec_{\mathcal{T}}$ between concept names by setting $A \prec_{\mathcal{T}} B$ if there exists an axiom of the form $A \equiv C$ or $A \sqsubseteq C$ in $\mathcal{T}$ such that $B$ occurs in $C$. A terminology $\mathcal{T}$ is called acyclic if the transitive closure $\prec_{\mathcal{T}}^{*}$ of $\prec_{\mathcal{T}}$ is irreflexive.

A signature $\Sigma$ is a finite subset of $\mathrm{N}_{\mathrm{C}} \cup \mathrm{N}_{\mathrm{R}}$. The signature $\operatorname{sig}(C)(\operatorname{sig}(\alpha)$, $\operatorname{sig}(\mathcal{T})$ ) of a concept $C$ (axiom $\alpha$, terminology $\mathcal{T}$ ) is the set of concept and role names which occur in $C(\alpha, \mathcal{T}$, respectively). If $\operatorname{sig}(C) \subseteq \Sigma$, we also call $C$ a $\Sigma$-concept and similarly for axioms and terminologies.

Definition 1 ( $\Sigma$-difference, $\Sigma$-entailment). Let $\mathcal{T}$ and $\mathcal{T}^{\prime}$ be terminologies and $\Sigma$ a signature. The $\Sigma$-difference, $\operatorname{Diff}_{\Sigma}\left(\mathcal{T}, \mathcal{T}^{\prime}\right)$, between $\mathcal{T}$ and $\mathcal{T}^{\prime}$ is defined as

$\operatorname{Diff}_{\Sigma}\left(\mathcal{T}, \mathcal{T}^{\prime}\right)=\left\{C \sqsubseteq D \mid \mathcal{T} \not \models C \sqsubseteq D\right.$ and $\mathcal{T}^{\prime} \models C \sqsubseteq D$ and $\left.\operatorname{sig}(C \sqsubseteq D) \subseteq \Sigma\right\}$.

$\mathcal{T} \Sigma$-entails $\mathcal{T}^{\prime}$ if, and only if, $\operatorname{Diff}_{\Sigma}\left(\mathcal{T}, \mathcal{T}^{\prime}\right)=\emptyset . \mathcal{T}$ and $\mathcal{T}^{\prime}$ are called $\Sigma$ inseparable if $\mathcal{T}$ and $\mathcal{T}^{\prime} \Sigma$-entail each other.

Example 1. Observe that, in some cases, $\operatorname{Diff}_{\Sigma}\left(\mathcal{T}, \mathcal{T}^{\prime}\right)$ contains concept implications of at least exponential size only, even for acyclic terminologies: let

$$
\begin{gathered}
\mathcal{T}=\left\{A_{1} \sqsubseteq F_{0}\right\} \cup\left\{F_{i} \sqsubseteq \exists r . F_{i+1} \sqcap \exists s . F_{i+1} \mid 0 \leq i<n\right\}, \\
\mathcal{T}^{\prime}=\left\{A_{0} \sqsubseteq B_{0}, A_{1} \equiv B_{n}\right\} \cup\left\{B_{i+1} \equiv \exists r . B_{i} \sqcap \exists s . B_{i} \mid 0 \leq i<n\right\},
\end{gathered}
$$

and $\Sigma=\left\{A_{0}, A_{1}, r, s\right\}$. Then $\mathcal{T}^{\prime}$ is not $\Sigma$-entailed by $\mathcal{T}$, and a minimal implication in $\operatorname{Diff}_{\Sigma}\left(\mathcal{T}, \mathcal{T}^{\prime}\right)$ is given by $C_{n} \sqsubseteq A_{1}$, where $C_{0}=A_{0}$ and $C_{i+1}=$ $\exists r . C_{i} \sqcap \exists s . C_{i}$, for $i \geq 0$. Clearly, $C_{n}$ is of exponential size. Observe, however, that if we use structure sharing and define the size of $C_{n}$ as the number of its subconcepts, then $C_{n}$ is only of polynomial size.

Observe that if $\mathcal{T} \Sigma$-entails $\mathcal{T}^{\prime}$, then $\mathcal{T} \Sigma^{\prime}$-entails $\mathcal{T}^{\prime}$ for any $\Sigma^{\prime}$ with $\Sigma^{\prime} \cap$ $\operatorname{sig}\left(\mathcal{T}^{\prime}\right) \subseteq \Sigma$. This follows immediately from the following interpolation result [16].

Theorem 1. $\mathcal{E} \mathcal{L}$ has the interpolation property, i.e., if $\mathcal{T} \models C \sqsubseteq D$, then there exists a finite set $\mathcal{T}_{0}$ of $C I$ s with $\operatorname{sig}\left(\mathcal{T}_{0}\right) \subseteq \operatorname{sig}(\mathcal{T}) \cap \operatorname{sig}(C \sqsubseteq D)$ such that $\mathcal{T} \models \mathcal{T}_{0}$ and $\mathcal{T}_{0} \models C \sqsubseteq D$. 


$$
\begin{gathered}
\overline{C \sqsubseteq C}(\mathrm{Ax}) \frac{C \sqsubseteq E}{C \sqsubseteq \top}(\mathrm{AxTop}) \frac{C \sqsubseteq E}{C \sqcap D \sqsubseteq E}(\mathrm{AndL}) \frac{D \sqsubseteq E}{C \sqcap D \sqsubseteq E} \text { (AndL2) } \\
\frac{C \sqsubseteq E \quad C \sqsubseteq D}{C \sqsubseteq D \sqcap E}(\mathrm{AndR}) \frac{C \sqsubseteq D}{\exists r . C \sqsubseteq \exists r . D}(\mathrm{Ex}) \\
\frac{C_{A} \sqsubseteq D}{A \sqsubseteq D}(\mathrm{DEFL}) \frac{D \sqsubseteq C_{A}}{D \sqsubseteq A}(\mathrm{DEFR}) \text { where } A \equiv C_{A} \in \mathcal{T} \\
\frac{C_{A} \sqsubseteq D}{A \sqsubseteq D}(\mathrm{PDEFL}) \text { where } A \sqsubseteq C_{A} \in \mathcal{T}
\end{gathered}
$$

Fig. 1. Gentzen-style proof system for $\mathcal{E} \mathcal{L}$ terminologies.

\section{Basic properties of $\mathcal{E} \mathcal{L}$}

We derive basic properties of $\mathcal{E} \mathcal{L}$ from the Gentzen-style sequent calculus of Hofmann [10]; see Figure $1 .^{1}$ The basic calculus of [10] considers $\mathcal{E} \mathcal{L}$ without the constant $T$ and for terminologies without primitive concept definitions. To take care of $T$, we have added the rule (AxTOP), and (PDEFL) is the rule representing axioms of the form $A \sqsubseteq C$. Cut-elimination, completeness, and correctness can now be proved by a straightforward extension of the proof in [10]. For a terminology $\mathcal{T}$ and concepts $C, D$, we write $\mathcal{T} \vdash C \sqsubseteq D$ iff there exists a proof of $C \sqsubseteq D$ in the calculus of Figure 1 .

Theorem 2 (Hofmann). For all terminologies $\mathcal{T}$ and concepts $C, D, \mathcal{T} \models C \sqsubseteq$ $D$ if, and only if, $\mathcal{T} \vdash C \sqsubseteq D$.

We apply this calculus to derive a description of the syntactic form of concepts $C$ such that $\mathcal{T} \models C \sqsubseteq D$, where $D$ is not equivalent to a conjunction. Call a concept name $A$ primitive in $\mathcal{T}$ if $A$ does not occur on the left hand side of an axiom in $\mathcal{T}$. Call $A$ pseudo-primitive in $\mathcal{T}$ if it is primitive in $\mathcal{T}$ or occurs on the left hand side of primitive concept definitions in $\mathcal{T}$. In what follows, we say that a concept $F$ is a conjunction of concepts if $F=\prod_{D \in X} D$, for a set $X$ of concepts. Any $D \in X$ is then called a conjunct of $F$ and, if $D$ is a concept name, then it is called an atomic conjunct of $F$. We sometimes write $D \in F$ instead of $D \in X$ and if $X$ is empty, then $F$ denotes the concept $\top$.

Lemma 1. Let $\mathcal{T}$ be a terminology and $C=F \sqcap \prod_{(r, D) \in Q} \exists$ r.D, where $F$ is a conjunction of concept names and $Q$ is a set of pairs $(r, D)$ in which $r$ is a role and $D$ a concept.

1. If $\mathcal{T} \models C \sqsubseteq A$ for an $A$ which is pseudo-primitive in $\mathcal{T}$, then $\mathcal{T} \models B \sqsubseteq A$, for some atomic conjunct $B$ of $F$.

\footnotetext{
${ }^{1}$ Alternatively, one could start from the model-theoretic analysis of $\mathcal{E} \mathcal{L}$ terminologies in [1].
} 
2. If $\mathcal{T} \models C \sqsubseteq \exists s . C_{0}$, then

$-\mathcal{T}=B \sqsubseteq \exists s . C_{0}$, for some atomic conjunct $B$ of $F$, or

- there exists $(r, D) \in Q$ such that $r=s$ and $\mathcal{T} \models D \sqsubseteq C_{0}$.

Proof. We use Theorem 2. Point 1. Let $\mathcal{T} \vdash C \sqsubseteq A$, where $A$ is pseudo-primitive in $\mathcal{T}$. Let $\mathcal{D}$ be a proof of $C \sqsubseteq A$. Note that, since $A$ is pseudo-primitive in $\mathcal{T}$, $\mathcal{D}$ may only end with one of $\mathrm{Ax}$, AndL1, AndL2, DeFL, or PDEFL. We show that the $\mathcal{T} \vdash B \sqsubseteq A$, for some conjunct $B$ of $F$, by induction on the number $n$ of conjuncts in $C$.

The base case of $n=1$ is trivial: $\mathcal{D}$ can only end with one of Ax, PDEFL, or DefL; so, $C$ is a concept name itself.

Assume $n>1$. Then $\mathcal{D}$ can only end with one of ANDL1 or ANDL2. In any case, there exists a conjunct $C^{\prime}$ of $C$ such that $\mathcal{T} \vdash C^{\prime} \sqsubseteq A$ and $C^{\prime}$ contains less conjuncts than $C$. By induction, there exists a concept name $B$ which is a conjunct in $C^{\prime}$ such that $\mathcal{T} \vdash B \sqsubseteq A$. Note now that $B$ is also a conjunct of $C$.

Point 2. Let $\mathcal{T} \vdash C \sqsubseteq \exists s . C_{0}$. Let $\mathcal{D}$ be a proof of $C \sqsubseteq \exists s . C_{0}$. Note that $\mathcal{D}$ may only end with one of Ax, AndL1, AndL2, DefL, PDefL, or Ex. We prove this part of lemma by induction on the number $n$ of conjuncts in $C$.

If $n=1$, the proof $\mathcal{D}$ can only end with one of Ax, DefL, PDEFL, or Ex. We have two subcases:

- $\mathcal{D}$ ends with DefL or PDefL. Then $C$ is $B$ for some concept name $B$ and $B \sqsubseteq \exists s . C_{0}$ is provable.

$-\mathcal{D}$ ends with Ax or Ex. Then $C$ is of the form $\exists$ s.D and $D \sqsubseteq C_{0}$ is provable.

The case $n>1$ can be proved by induction similarly to Point 1 above.

We apply Lemma 1 to show that if $\mathcal{T}$ does not $\Sigma$-entail $\mathcal{T}^{\prime}$, then there exists $C \sqsubseteq D \in \operatorname{Diff}_{\Sigma}\left(\mathcal{T}, \mathcal{T}^{\prime}\right)$ such that $C$ or $D$ is a concept name.

Lemma 2. Let $\mathcal{T}$ and $\mathcal{T}^{\prime}$ be terminologies and $\Sigma$ a signature. If $C \sqsubseteq D \in$ $\operatorname{Diff}_{\Sigma}\left(\mathcal{T}, \mathcal{T}^{\prime}\right)$, then there exist subconcepts $C^{\prime}$ and $D^{\prime}$ of $C$ and $D$, respectively, such that $C^{\prime} \sqsubseteq D^{\prime} \in \operatorname{Diff}_{\Sigma}\left(\mathcal{T}, \mathcal{T}^{\prime}\right)$ and $C^{\prime} \sqsubseteq D^{\prime}$ is of the form $A \sqsubseteq \exists r . D_{0}$ or $C_{0} \sqsubseteq A$, where $A$ is a concept name.

Proof. Let $C \sqsubseteq D \in \operatorname{Diff}_{\Sigma}\left(\mathcal{T}, \mathcal{T}^{\prime}\right)$. Then $D \neq \top$ because otherwise $\mathcal{T}=C \sqsubseteq \top$. If $D=D_{1} \sqcap D_{2}$, then one of $C \sqsubseteq D_{i}, i=1,2$, is in the $\Sigma$-difference. If $D=\exists r . D_{1}$ then, by Lemma 1 , either there exists a subconcept $A$ of $C, A$ a concept name, such that $A \sqsubseteq D$ is in the $\Sigma$-difference, or there exists a subconcept $\exists r . C_{1}$ of $C$, such that $C_{1} \sqsubseteq D_{1}$ is in the $\Sigma$-difference. Simplify $C \sqsubseteq D$ until none of these simplification rules is applicable. The resulting CI is as required.

\section{Deciding $\Sigma$-entailment: theory}

By Lemma 2, to decide $\Sigma$-entailment, it is sufficient to decide whether the set $\operatorname{Diff}_{\Sigma}\left(\mathcal{T}, \mathcal{T}^{\prime}\right)$ contains $\Sigma$-implications of the form $C \sqsubseteq A$ or $A \sqsubseteq D$, where $A$ is a concept name. The latter problem is decidable in polynomial time already for 


$$
\begin{aligned}
& \text { For } A \in \mathrm{N}_{\mathrm{C}} \text {, } \\
& \text { - if } A \text { is pseudo-primitive in } \mathcal{T} \text {, then } \\
& \text { noimply }_{\mathcal{T}, \Sigma}(A)=\left\{\xi_{A}\right\}, \quad \text { Noimply }_{\mathcal{T}, \Sigma}(A)=\left\{\xi_{A} \sqsubseteq \prod_{A^{\prime} \in\left(\Sigma \backslash \operatorname{pre}_{\mathcal{T}}^{\Sigma}(A)\right)} A^{\prime} \sqcap \mathrm{All}_{\Sigma}\right\} ; \\
& \text { - if } A \text { is conjunctive in } \mathcal{T} \text { and } A \equiv F \in \mathcal{T} \text {, then } \\
& \text { noimply }_{\mathcal{T}, \Sigma}(A)=\left\{\xi_{B} \mid B \in F\right\}, \quad \text { Noimply }_{\mathcal{T}, \Sigma}(A)=\emptyset \\
& \text { - if } A \equiv \exists r . B \in \mathcal{T} \text {, then noimply } \mathcal{T}, \Sigma(A)=\left\{\xi_{A}\right\} \text { and Noimply } \mathcal{T}, \Sigma_{\mathcal{T}}(A)=\left\{\alpha_{A}\right\} \text {, where } \\
& \alpha_{A}=\xi_{A} \sqsubseteq\left(\prod_{A^{\prime} \in\left(\Sigma \backslash \operatorname{pre} \frac{\Sigma}{T}(A)\right)} A^{\prime}\right) \sqcap\left(\prod_{r \neq s \in \Sigma} \exists s .\left(\prod_{A^{\prime} \in \Sigma} A^{\prime} \sqcap \mathrm{All}_{\Sigma}\right)\right) \sqcap \prod_{\xi \in \text { noimply }_{\mathcal{T}, \Sigma}(B)} \exists r . \xi .
\end{aligned}
$$

Fig. 2. Computing Noimply $_{\mathcal{T}, \Sigma}(A)$ and noimply ${ }_{\mathcal{T}, \Sigma}(A)$

general $\mathcal{E} \mathcal{L}$-TBoxes [13]. So, in what follows we concentrate on $\Sigma$-implications of the form $C \sqsubseteq A$. We first transform $\mathcal{T}$ into a normalised terminology. A concept name $A$ is called non-conjunctive in $\mathcal{T}$ if it is pseudo-primitive in $\mathcal{T}$ or has a definition of the form $A \equiv \exists r . C \in \mathcal{T}$. Otherwise $A$ is called conjunctive in $\mathcal{T}$. A terminology $\mathcal{T}$ is normalised if it consists of axioms of the following form:

- $A \equiv \exists r . B$ or $A \sqsubseteq \exists r . B$, where $B$ is a concept name;

- $A \equiv F$ or $A \sqsubseteq F$, where $F$ is a (possibly empty) conjunction of concept names such that every conjunct $B$ of $F$ is non-conjunctive in $\mathcal{T}$.

Normalised terminologies in the sense defined above are a minor modification of normalised terminologies as defined in [1]. Say that two interpretations $\mathcal{I}$ and $\mathcal{J}$ coincide on a signature $\Sigma$, in symbols $\left.\mathcal{I}\right|_{\Sigma}=\left.\mathcal{J}\right|_{\Sigma}$, if $\Delta^{\mathcal{I}}=\Delta^{\mathcal{J}}$ and $X^{\mathcal{I}}=X^{\mathcal{J}}$ for all $X \in \Sigma$.

Lemma 3. For every terminology $\mathcal{T}$, one can construct in polynomial time a normalised terminology $\mathcal{T}^{\prime}$ of polynomial size in $|\mathcal{T}|$ such that $\operatorname{sig}(\mathcal{T}) \subseteq \operatorname{sig}\left(\mathcal{T}^{\prime}\right)$, $\mathcal{T}^{\prime}=\mathcal{T}$, and for every model $\mathcal{I}$ of $\mathcal{T}$ there exists a model $\mathcal{J}$ of $\mathcal{T}^{\prime}$ which coincides with $\mathcal{I}$ on $\Sigma$. Moreover, $\mathcal{T}^{\prime}$ is acyclic if $\mathcal{T}$ is acyclic.

The proof is a straightforward modification of the proof in [1], see also Appendix. From now on we will work with normalised terminologies only.

Intuitively, to decide whether there exists $C \sqsubseteq A \in \operatorname{Diff}_{\Sigma}\left(\mathcal{T}, \mathcal{T}^{\prime}\right)$, we want to construct the most specific $\Sigma$-concept $C_{A}$ such that $\mathcal{T} \not \models C_{A} \sqsubseteq A$. Then there exists some $\Sigma$-concept $C$ such that $C \sqsubseteq A \in \operatorname{Diff}_{\Sigma}\left(\mathcal{T}, \mathcal{T}^{\prime}\right)$ if, and only if, $\mathcal{T}^{\prime} \models C_{A} \sqsubseteq A$. Unfortunately, most specific $\Sigma$-concepts with this property do not always exist and, therefore (and also to enable structure sharing), we use an additional terminology. We use the following sets and axiom:

$-\Sigma^{\text {fresh }}=\left\{\right.$ All $\left._{\Sigma}\right\} \cup\left\{\xi_{A} \mid A \in \mathrm{N}_{C}\right.$ non-conjunctive in $\left.\mathcal{T}\right\}$ is a set of fresh concept names not occurring in $\Sigma \cup \operatorname{sig}(\mathcal{T})$; 
- $\alpha$ denotes the concept inclusion $\mathrm{All}_{\Sigma} \sqsubseteq \prod_{r \in \Sigma} \exists r .\left(\prod_{A^{\prime} \in \Sigma} A^{\prime} \sqcap \mathrm{All} \mathrm{l}_{\Sigma}\right)$;

- $\operatorname{pre}_{\mathcal{T}}^{\Sigma}(A)=\{B \in \Sigma \mid \mathcal{T}=B \sqsubseteq A\}$, for $A \in \mathrm{N}_{\mathrm{C}}$. These sets can be computed in polynomial time [1].

Theorem 3. Let $\mathcal{T}$ be a normalised terminology and $\Sigma$ a signature. The terminologies Noimply $_{\mathcal{T}, \Sigma}(A)$ and sets of concepts names noimply $\operatorname{l}_{\mathcal{T}, \Sigma}(A)$ are constructed, in polynomial time, in Figure 2. Set

$$
\text { Noimply }_{\mathcal{T}, \Sigma}=\{\alpha\} \cup \bigcup_{A \in \Sigma \cup \operatorname{sig}(\mathcal{T})} \operatorname{Noimply}_{\mathcal{T}, \Sigma}(A) .
$$

The following conditions are equivalent, for every concept name $A \in \Sigma \cup \operatorname{sig}(\mathcal{T})$ and terminology $\mathcal{T}^{\prime}$ with $\operatorname{sig}\left(\mathcal{T}^{\prime}\right) \cap \Sigma^{\text {fresh }}=\emptyset$ :

- there exists a $\Sigma$-concept $C$ with $\mathcal{T}^{\prime} \models C \sqsubseteq A$ and $\mathcal{T} \not \models C \sqsubseteq A$;

- $\mathcal{T}^{\prime} \cup$ Noimply $_{\mathcal{T}, \Sigma} \models \xi \sqsubseteq A$, for some $\xi \in$ noimply $_{\mathcal{T}, \Sigma}(A)$.

Observe that, in Theorem 3, Noimply $\mathcal{T}, \Sigma_{\text {and }}$ noimply $\operatorname{li}_{\mathcal{T}, \Sigma}(A)$ do not depend on $\mathcal{T}^{\prime}$. Thus, once they have been constructed, they can be used to check the existence of concept implications $C \sqsubseteq A \in \operatorname{Diff}_{\Sigma}\left(\mathcal{T}, \mathcal{T}^{\prime}\right)$ for arbitrary terminologies $\mathcal{T}^{\prime}$. It is worth noting as well that the proof of Theorem 3 will show that the result holds for arbitrary general TBoxes $\mathcal{T}^{\prime}$ formulated in description logics which are fragments of first-order logic, and, indeed, for $\mathcal{T}^{\prime}$ any first-order theory. In this case, Theorem 3 provides a reduction of checking whether there exists $C \sqsubseteq A \in \operatorname{Diff}_{\Sigma}\left(\mathcal{T}, \mathcal{T}^{\prime}\right)$ to deduction in the language of $\mathcal{T}^{\prime}$.

Example 2. Let $\mathcal{T}=\{A \equiv \exists r . B, B \equiv \exists r . A\}$ and $\Sigma=\{r, A, B\}$. Then we have noimply $_{\mathcal{T}, \Sigma}(A)=\left\{\xi_{A}\right\}$ and Noimply $\mathcal{T}, \Sigma=\left\{\xi_{A} \sqsubseteq B \sqcap \exists r . \xi_{B}, \xi_{B} \sqsubseteq A \sqcap \exists r . \xi_{A}\right\}$. Intuitively, $\left\{\xi_{A}\right\} \cup$ Noimply $_{\mathcal{T}, \Sigma}$ stands for the "infinitary" most specific $\Sigma$-concept not subsumed by $A$ relative to $\mathcal{T}$.

In the remainder of this section we prove Theorem 3. To this end, we first prove an "infinitary" version of Theorem 3 by associating with every concept name $A$ a sequence noimply ${ }_{\mathcal{T}, \Sigma}^{n}(A), n \geq 0$, of sets of $\Sigma$-concepts such that the following holds:

C1. $\mathcal{T} \not \forall C \sqsubseteq A$ for all $n \geq 0$ and $C \in$ noimply $_{\mathcal{T}, \Sigma}^{n}(A)$.

C2. For all $\Sigma$-concepts $D$, if $\mathcal{T} \forall \forall D \sqsubseteq A$, then $\models C \sqsubseteq D$ for some $C \in$ noimply $\tilde{T}, \Sigma^{n}(A)$, where $n$ is the role-depth $\operatorname{depth}(D)$ of $D$ (i.e., the number of nestings of existential restrictions in $D) .^{2}$

The sets noimply ${ }_{\mathcal{T}, \Sigma}^{n}(A)$ are defined in Figure 3 . Observe that noimply ${ }_{\mathcal{T}, \Sigma}^{n}(A)$ is well-defined because in the definition $A \equiv F \in \mathcal{T}$ of a conjunctive concept name $A$ no conjunctive concept name occurs. This observation will also be used in the inductive proofs below.

${ }^{2}$ More precisely $\operatorname{depth}(A)=0, \operatorname{depth}\left(C_{1} \sqcap C_{2}\right)=\max \left\{\operatorname{depth}\left(C_{1}\right), \operatorname{depth}\left(C_{2}\right)\right\}$, and $\operatorname{depth}(\exists r . D)=\operatorname{depth}(D)+1$. 


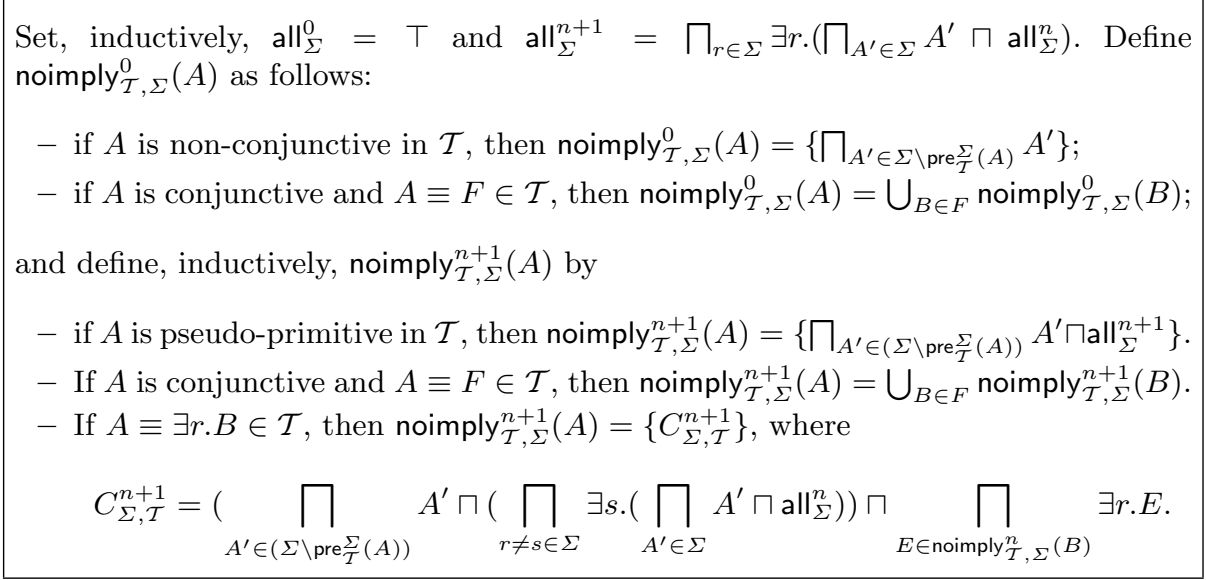

Fig. 3. Computing noimply ${ }_{\mathcal{T}, \Sigma}^{n}(A)$

Example 3. For the terminology $\mathcal{T}$ and signature $\Sigma$ from Example 2, we have noimply $_{\mathcal{T}, \Sigma}^{0}(A)=\{B\}$, noimply ${ }_{\mathcal{T}, \Sigma}^{1}(A)=\{B \sqcap \exists r . A\}$, noimply ${ }_{\mathcal{T}, \Sigma}^{2}(A)=\{B \sqcap$ $\exists r .(A \sqcap \exists r . B)\}$, etc. Thus, intuitively, noimply $\mathcal{T}_{, \Sigma}^{n}(A)$ is the unfolding up to depth $n$ of $\xi_{A}$ relative to Noimply $_{\mathcal{T}, \Sigma}$.

Lemma 4. Let $\mathcal{T}$ be a normalised terminology, signature $\Sigma$, and $A \in \mathrm{N}_{\mathrm{C}}$. The sets noimply ${ }_{\mathcal{T}, \Sigma}^{n}(A)$ satisfy conditions $\boldsymbol{C 1}$ and $\boldsymbol{C} 2$ above.

Proof. C1. Assume first that $A$ is pseudo-primitive in $\mathcal{T}$. Then noimply ${ }_{\mathcal{T}, \Sigma}^{n}(A)$ consists of $C=\prod_{A^{\prime} \in\left(\Sigma \backslash \operatorname{pre}_{\mathcal{T}}^{\Sigma}(A)\right)} A^{\prime} \sqcap$ all ${ }_{\Sigma}^{n}$. By Lemma $1, \mathcal{T} \not \models C \sqsubseteq A$ because the only atomic conjuncts of $C$ are in $\Sigma \backslash \operatorname{pre}_{\mathcal{T}}^{\Sigma}(A)$.

We now prove $\mathbf{C} \mathbf{1}$ for concept names $A$ which are not pseudo-primitive in $\mathcal{T}$. The proof is by induction on $n$. For $n=0$ and $A \equiv \exists r . B \in \mathcal{T}$ the claim follows again from Lemma 1 and the observation that $B^{\prime} \in \operatorname{pre}_{\mathcal{T}}^{\Sigma}(A)$ if, and only if, $\mathcal{T} \models B^{\prime} \sqsubseteq \exists r . B$. For $n=0$ and $A$ conjunctive with $A \equiv F \in \mathcal{T}$, C1 follows since it has been proved for all conjuncts of $F$ and $\mathcal{T} \not \models C \sqsubseteq A$ if, and only if, there exists an atomic conjunct $B$ of $F$ such that $\mathcal{T} \not \models C \sqsubseteq B$.

For the induction step, assume $\mathbf{C} 1$ has been proved for $n \geq 0$.

Let $A \equiv \exists r . B \in \mathcal{T}$ and let $C_{\mathcal{T}, \Sigma}^{n+1}$ be the only element of noimply $\mathcal{T}_{\mathcal{T}, \Sigma}^{n+1}(A)$. Assume $\mathcal{T} \models C_{\mathcal{T}, \Sigma}^{n+1} \sqsubseteq A$. By Lemma 1 there are two possibilities:

$-\mathcal{T} \models \prod_{A^{\prime} \in\left(\Sigma \backslash \operatorname{pre}_{\mathcal{T}}^{\Sigma}(A)\right)} A^{\prime} \sqsubseteq \exists r$. B. This is excluded, by Lemma 1 .

- There exists $E \in$ noimply $_{\mathcal{T}, \Sigma}^{n}(B)$ such that $\mathcal{T} \models E \sqsubseteq B$. This is excluded by the induction hypothesis.

We have derived a contradiction. The case $A \equiv F \in \mathcal{T}, A$ conjunctive in $\mathcal{T}$, is considered similarly to the case $n=0$ and left to the reader.

C2. Let $n=0$ and assume first that $A$ is non-conjunctive. Let $D$ be a $\Sigma$ concept with $\operatorname{depth}(D)=0$ and $\mathcal{T} \not \models D \sqsubseteq A$. Then all conjuncts of $D$ are in $\Sigma \backslash$ 
$\operatorname{pre}_{\mathcal{T}}^{\Sigma}(A)$ and we obtain $\models \prod_{A^{\prime} \in \Sigma \backslash \operatorname{pre}_{\mathcal{T}}^{\Sigma}(A)} A^{\prime} \sqsubseteq D$. Now assume $A$ is conjunctive in $\mathcal{T}$ and $A \equiv F \in \mathcal{T}$. Let $D$ be a $\Sigma$-concept with $\operatorname{depth}(D)=0$ and $\mathcal{T} \not=$ $D \sqsubseteq A$. Then $\mathcal{T} \not \models D \sqsubseteq B$, for some conjunct $B$ of $F$. By induction, $\models C \sqsubseteq D$ for the (unique) $C \in$ noimply $_{\mathcal{T}, \Sigma}^{0}(B)$, and therefore $\models C \sqsubseteq D$ for some $C \in$ noimply $_{\mathcal{T}, \Sigma}^{0}(A)$.

For the induction step, assume that $\mathbf{C 2}$ has been shown for $n$. Let $D$ be a $\Sigma$-concept with $\mathcal{T} \not \models D \sqsubseteq A$ and $\operatorname{depth}(D)=n+1$.

(a) Let $A$ be pseudo-primitive in $\mathcal{T}$. Then the atomic conjuncts of $D$ are included in $\Sigma \backslash \operatorname{pre}_{\mathcal{T}}^{\Sigma}(A)$. Now $\models C \sqsubseteq D$ follows immediately for $C=\prod_{A^{\prime} \in \Sigma \backslash \operatorname{pre}_{\mathcal{T}}^{\Sigma}(A)} A^{\prime} \sqcap$ all $\Sigma_{\Sigma}^{n+1}$.

(b) Let $A \equiv \exists r . B \in \mathcal{T}$. Let $C_{\mathcal{T}, \Sigma}^{n+1}$ be the only element of noimply $\operatorname{T}_{\mathcal{T}, \Sigma}^{n+1}(A)$ and assume

$$
D=\prod_{B \in Q_{0}} B \sqcap \prod_{\left(s, D^{\prime}\right) \in Q_{1}} \exists s \cdot D^{\prime} .
$$

Then $Q_{0} \subseteq \Sigma \backslash \operatorname{pre}_{\mathcal{T}}^{\Sigma}(A)$. Hence, $\models C_{\mathcal{T}, \Sigma}^{n+1} \sqsubseteq \prod_{B \in Q_{0}} B$. Now consider a conjunct $\exists s . D^{\prime}$ of $D$. There are two cases:

$-s \neq r$. Then, clearly, $\models C_{\mathcal{T}, \Sigma}^{n+1} \sqsubseteq \exists s . D^{\prime}$.

$-s=r$. It is enough to show that there exists $E \in$ noimply $_{\mathcal{T}, \Sigma}^{n}(B)$ such that $\models E \sqsubseteq D^{\prime}$. Suppose there does not exist such an $E$. Then, by $\mathrm{IH}$, $\mathcal{T}=D^{\prime} \sqsubseteq B$. But this contradicts $\mathcal{T} \not \mid C_{\mathcal{T}, \Sigma}^{n+1} \sqsubseteq \exists r . B$.

(c) $A$ is conjunctive in $\mathcal{T}$ and $A \equiv F \in \mathcal{T}$. This case is left to the reader.

Corollary 1. For all terminologies $\mathcal{T}^{\prime}$ and $A \in \mathrm{N}_{\mathrm{C}}$ the following are equivalent:

1. there exists a $\Sigma$-concept $C$ such that $\mathcal{T} \not \models C \sqsubseteq A$ and $\mathcal{T}^{\prime} \models C \sqsubseteq A$;

2. there exists $n \geq 0$ and $C \in$ noimply $_{\mathcal{T}, \Sigma}^{n}(A)$ such that $\mathcal{T}^{\prime} \models C \sqsubseteq A$.

Proof. The direction from Point 2 to Point 1 follows immediately from C1. Conversely, assume that there exists a $\Sigma$-concept $C$ such that $\mathcal{T}^{\prime} \models C \sqsubseteq A$ and $\mathcal{T} \not \models C \sqsubseteq A$. By $\mathbf{C 1}$ and $\mathbf{C 2}$, there exist $n$ and $C^{\prime} \in$ noimply $_{\mathcal{T}, \Sigma}^{n}(A)$ with $\models C^{\prime} \sqsubseteq C$ and $\mathcal{T} \not \models C^{\prime} \sqsubseteq A$. Then $\mathcal{T}^{\prime} \models C^{\prime} \sqsubseteq A$.

In contrast to the formulation of Theorem 3, Corollary 1 does not provide us with a polynomial time algorithm. First, no upper bound on $n$ is given and, second, the concepts in noimply ${ }_{\mathcal{T}, \Sigma}^{n}(A)$ are of exponential size in $n$. Example 1 is easily extended so as to show that this is unavoidable: one can construct a terminology $\mathcal{T}$ and a sequence of terminologies $\mathcal{T}_{n}^{\prime}$ such that in minimal implications in $\operatorname{Diff}_{\Sigma}\left(\mathcal{T}, \mathcal{T}^{\prime}\right)$ of the form $C_{n} \sqsubseteq A$ the concept $C_{n}$ has at least depth $n$ and is of size $2^{n}$. However, Theorem 3 is now an immediate consequence of the following lemma and Corollary 1.

Lemma 5. Let $\mathcal{T}^{\prime}$ be a terminology such that $\operatorname{sig}\left(\mathcal{T}^{\prime}\right) \cap \Sigma^{\text {fresh }}=\emptyset$ and $A \in$ $\operatorname{sig}(\mathcal{T}) \cup \Sigma$. Then the following conditions are equivalent:

1. $\mathcal{T}^{\prime} \cup$ Noimply $_{\mathcal{T}, \Sigma} \models \xi \sqsubseteq A$, for some $\xi \in$ noimply $_{\mathcal{T}, \Sigma}(A)$; 
2. $\mathcal{T}^{\prime} \models C \sqsubseteq A$, for some $n \geq 0$ and $C \in$ noimply $_{\mathcal{T}, \Sigma}^{n}(A)$.

Proof. Point 2 implies Point 1. For concept names $A$ which are non-conjunctive in $\mathcal{T}$ this follows because Noimply $\mathcal{T}, \Sigma_{,} \mid=\xi_{A} \sqsubseteq C$ for the only element $C$ of noimply ${ }_{\mathcal{T}, \Sigma}^{n}(A)$. The conjunctive case follows by induction.

Point 1 implies Point 2 is proved by a compactness argument. Intuitively, if $\mathcal{T}^{\prime} \cup \bigcup_{n>0}$ noimply $_{\mathcal{T}, \Sigma}^{n}(A) \not \models A$, then $\mathcal{T}^{\prime} \cup$ Noimply $_{\mathcal{T}, \Sigma} \not \models \xi \sqsubseteq A$, for all $\xi \in$ noimply $_{\mathcal{T}, \Sigma}(A)$. However, to prove this, one has to re-construct the concepts noimply $_{\mathcal{T}, \Sigma}^{n}(A)$; details are provided in the Appendix.

\section{Practical algorithm and system}

We have seen above that the sets

- $\operatorname{DiffR}_{\Sigma}\left(\mathcal{T}, \mathcal{T}^{\prime}\right)$ consisting of all $A \in \Sigma$ such that there is a $\Sigma$-concept $C$ with $\mathcal{T} \not \models C \sqsubseteq A$ and $\mathcal{T}^{\prime} \models C \sqsubseteq A$, and

- DiffL $L_{\Sigma}\left(\overline{\mathcal{T}}, \mathcal{T}^{\prime}\right)$ consisting of all $A \in \Sigma$ such that there is a $\Sigma$-concept $C$ with $\mathcal{T} \not \models A \sqsubseteq C$ and $\mathcal{T}^{\prime} \models A \sqsubseteq C$

can be computed in polynomial time and can be regarded, by Lemma 2 , as an informative approximation of the logical difference between $\mathcal{T}$ and $\mathcal{T}^{\prime}$ w.r.t. $\Sigma$.

Computing both sets for large terminologies and signatures $\Sigma$ using a direct implementation of the algorithm described above will fail. Considering that state of the art description logic reasoners [2] take about 15 minutes to classify the SNOMED CT terminology [17], the reduction to reasoning given in Section 4 is impractical for large terminologies and signatures of reasonable size (the terminology Noimply $_{\mathcal{T}, \Sigma}$ contains huge conjunctions of $\Sigma$-concept names). We now discuss the implementation of the algorithms above in the system CEX for acyclic terminologies using a dynamic programming approach.

Let $\mathcal{T}$ and $\mathcal{T}^{\prime}$ be acyclic terminologies and $\Sigma$ a signature. For expositional reasons, we assume that $\Sigma \subseteq \operatorname{sig}\left(\mathcal{T}^{\prime}\right) \subseteq \operatorname{sig}(\mathcal{T})$. This is justified because we can add $A \sqsubseteq \top$ to $\mathcal{T}^{\prime}$, for all $A \in \Sigma \backslash \operatorname{sig}\left(\mathcal{T}^{\prime}\right)$, and $A \sqsubseteq \top$ to $\mathcal{T}$, for all $A \in$ $\left(\Sigma \cup \operatorname{sig}\left(\mathcal{T}^{\prime}\right)\right) \backslash \operatorname{sig}(\mathcal{T})$. We describe the algorithm computing $\operatorname{DiffR}_{\Sigma}$, the algorithm computing Diff $\mathrm{L}_{\Sigma}$ is discussed in the technical report. We assume that $\mathcal{T}$ and $\mathcal{T}^{\prime}$ are fully classified and the result of the classification is kept in a table, so, given two concept names $A$ and $B$, it takes constant time to find out whether $\mathcal{T} \models A \sqsubseteq B$ (likewise, if $\mathcal{T}^{\prime} \models A \sqsubseteq B$ ). Now the algorithm computing DiffR ${ }_{\Sigma}$ works by induction on concept definitions and marks, recursively, every $E \in$ $\operatorname{sig}\left(\mathcal{T}^{\prime}\right)$, starting with pseudo-primitive ones, with members of $\Xi=\left\{\xi_{A} \mid A \in\right.$ $\operatorname{sig}(\mathcal{T})$ non-conjunctive in $\mathcal{T}$ \} in such a way that

(†) $E \in \operatorname{sig}\left(\mathcal{T}^{\prime}\right)$ is marked with $\xi$ if, and only if, $\mathcal{T}^{\prime} \cup$ Noimply $_{\mathcal{T}, \Sigma} \not \models \xi \sqsubseteq E$.

Then $A \in \Sigma$ is not marked with $\xi \in$ noimply $_{\mathcal{T}, \Sigma}(A)$ if, and only if, $\mathcal{T}^{\prime} \cup$ Noimply $_{\mathcal{T}, \Sigma} \models \xi \sqsubseteq A$. If this happens to be the case for some $\xi \in$ noimply $_{\mathcal{T}, \Sigma}(A)$, then $A$ is included in $\operatorname{DiffR}_{\Sigma}\left(\mathcal{T}, \mathcal{T}^{\prime}\right)$ (Theorem 3).

In order to define the marking, set $\operatorname{pre}_{\mathcal{T}}^{\Sigma}\left(\xi_{A}\right)=\operatorname{pre}_{\mathcal{T}}^{\Sigma}(A)$, for $A \in \operatorname{sig}(\mathcal{T})$ non-conjunctive in $\mathcal{T}$. Now mark $E \in \operatorname{sig}\left(\mathcal{T}^{\prime}\right)$ as follows: 
1. If $E$ is pseudo-primitive in $\mathcal{T}^{\prime}$, then it is marked with all $\xi \in \Xi$ such that $\operatorname{pre}_{\mathcal{T}^{\prime}}^{\Sigma}(E) \subseteq \operatorname{pre}_{\mathcal{T}}^{\Sigma}(\xi)$

2. If $E \equiv E_{1} \sqcap \ldots \sqcap E_{k} \in \mathcal{T}^{\prime}$, then it is marked with all $\xi \in \Xi$ such that at least one of $E_{1}, \ldots, E_{k}$ is marked with $\xi$;

3. If $E \equiv \exists r . E^{\prime} \in \mathcal{T}^{\prime}$ and

(a) if $r \notin \Sigma$ or $\mathcal{T}^{\prime} \cup\{\alpha\} \not \forall\left(\prod_{A^{\prime} \in \Sigma} A^{\prime} \sqcap\right.$ All $\left.{ }_{\Sigma}\right) \sqsubseteq E^{\prime}$, then $E$ is marked with all $\xi \in \Xi$ such that $\operatorname{pre}_{\mathcal{T}^{\prime}}^{\Sigma}(E) \subseteq \operatorname{pre}_{\mathcal{T}}^{\Sigma}(\xi)$;

(b) if $r \in \Sigma$ and $\mathcal{T}^{\prime} \cup\{\alpha\} \models\left(\prod_{A^{\prime} \in \Sigma} A^{\prime} \sqcap\right.$ All $\left.{ }_{\Sigma}\right) \sqsubseteq E^{\prime}$ then $E$ is marked with all $\xi_{A} \in \Xi$ such that

- $A \equiv \exists r . A^{\prime}$ in $\mathcal{T}$ and, for all $\xi^{\prime} \in$ noimply $_{\mathcal{T}, \Sigma}\left(A^{\prime}\right), E^{\prime}$ is marked with $\xi^{\prime}$ and

$-\operatorname{pre}_{\mathcal{T}^{\prime}}^{\Sigma}(E) \subseteq \operatorname{pre}_{\mathcal{T}}^{\Sigma}\left(\xi_{A}\right)$.

Using Theorem 3 and Lemma 5, one can prove that the defined marking has property ( $\dagger$ ), see Appendix. While the condition $\mathcal{T}^{\prime} \cup\{\alpha\} \models\left(\prod_{A^{\prime} \in \Sigma} A^{\prime} \sqcap A \|_{\Sigma}\right) \sqsubseteq$ $E$ can be checked directly, this requires operating concepts of large size for large $\Sigma$ 's. So, instead we use the following criterion: we may assume that $\mathcal{T}$ contains a definition $A \sqsubseteq \top$ such that $A \notin \operatorname{sig}\left(\mathcal{T}^{\prime}\right)$ and $A$ does not occur elsewhere in $\mathcal{T}$. Then it follows from the definitions that $\mathcal{T}^{\prime} \cup\{\alpha\} \models\left(\prod_{A^{\prime} \in \Sigma} A^{\prime} \sqcap \mathrm{All}_{\Sigma}\right) \sqsubseteq E$ if, and only if, $E$ is not marked with $\xi_{A}$.

Then all $\mathcal{T}^{\prime}$ concept names can be marked in $\mathcal{O}\left(|\mathcal{T}| \times\left|\mathcal{T}^{\prime}\right| \times|\Sigma|+T^{\prime}\right)$ time, where $T^{\prime}$ is the time taken to fully classify $\mathcal{T}^{\prime}$. Overall, checking the $\Sigma$-entailment takes $\mathcal{O}\left(|\mathcal{T}| \times\left|\mathcal{T}^{\prime}\right| \times|\Sigma|+T+T^{\prime}\right)$ time and $\mathcal{O}\left(|\mathcal{T}| \times\left|\mathcal{T}^{\prime}\right| \times|\Sigma|\right)$ space. It should be noted that in our implementation this theoretical upper bound is often not reached due to the use of hash tables and structure sharing.

\section{Experimental evaluation}

CEX is an OCaml program [4]. For the experiments, we use two versions of SnOmed CT: one dated 09 February 2005 (SM-05) and the other 30 December 2006 (SM-06) and having 379691 and 389472 axioms, respectively. As CEX currently accepts acyclic $\mathcal{E} \mathcal{L}$-terminologies only, the role inclusions of SNOMED CT are not taken into account. The tests have been carried out on a standard PC: Intel $囚$ Core ${ }^{\mathrm{TM}} 2 \mathrm{CPU}$ at $2.13 \mathrm{GHz}$ and $3 \mathrm{~GB}$ of RAM.

Logical difference between SM-05 and SM-06. Table 1 shows the average sizes of the lists DiffL ${ }_{\Sigma}(\mathrm{SM}-05, \mathrm{SM}-06)$ and DiffR ${ }_{\Sigma}(\mathrm{SM}-05, \mathrm{SM}-06)$ for 20 randomly generated signatures $\Sigma \subseteq \operatorname{sig}(\mathrm{SM}-05) \cap \operatorname{sig}(\mathrm{SM}-06)$ containing 100, 1000 , etc. concept names and 0,20 , or 40 role names. ${ }^{3}$ The execution time and memory consumption of CEX when computing these lists vary from 477 to 596 seconds and from 1393 to 1496 MByte, respectively. The numbers show that there is a huge difference between SM-05 and SM-06. Also, adding a role name to the signature has a larger impact on the number of differences than adding a concept name.

\footnotetext{
3 There are 50 role names in sig $(\mathrm{SM}-05) \cap \operatorname{sig}(\mathrm{SM}-06)$.
} 


\begin{tabular}{|c||c|c||c|c||c|c|}
\hline \multicolumn{2}{|c||}{$\left|\Sigma \cap \mathrm{N}_{\mathrm{C}}\right|$} & \multicolumn{2}{|c||}{$\left|\Sigma \cap \mathrm{N}_{\mathrm{R}}\right|=0$} & \multicolumn{2}{|c||}{$\left|\Sigma \cap \mathrm{N}_{\mathrm{R}}\right|=20$} & \multicolumn{2}{|c|}{$\left|\Sigma \cap \mathrm{N}_{\mathrm{R}}\right|=40$} \\
\cline { 2 - 7 } & $\mid$ diffL $_{\Sigma} \mid$ & $\mid$ diffR $_{\Sigma} \mid$ & $\mid$ diffL $_{\Sigma} \mid$ & $\mid$ diffR $_{\Sigma} \mid$ & $\mid$ diffL $_{\Sigma} \mid$ & $\left|\operatorname{diffR}_{\Sigma}\right|$ \\
\hline \hline 100 & 0.10 & 0.10 & 0.90 & 0.15 & 2.95 & 0.20 \\
\hline 1000 & 2.35 & 2.15 & 15.55 & 2.95 & 28.85 & 3.75 \\
\hline 10000 & 155.35 & 125.35 & 257.35 & 136.20 & 514.10 & 209.90 \\
\hline 100000 & 11795.90 & 4108.60 & 12954.45 & 4358.30 & 14942.55 & 6823.60 \\
\hline
\end{tabular}

Table 1. Computing logical difference with CEX: Diff ${ }_{\Sigma}(\mathrm{SM}-05, \mathrm{SM}-06)$

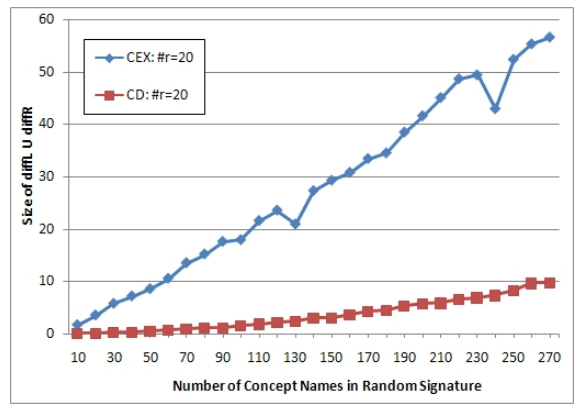

(a) Number of differences

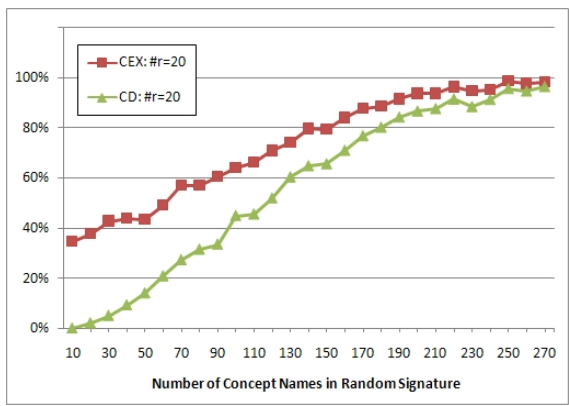

(b) Proportion of detected differences

Fig. 4. Comparison of CEX and classification-based approach

Comparison with the classification approach. We compare the size of $\operatorname{Diff}_{\Sigma} \cup$ DiffR $_{\Sigma}$ as computed by CEX with the number of concept names in $\Sigma$ for which there is a difference in the class hierarchy restricted to $\Sigma$; i.e., the set of $A \in \Sigma$ such that there exists $B \in \Sigma$ such that $A \sqsubseteq B \in \operatorname{Diff}_{\Sigma}$ or $B \sqsubseteq A \in \operatorname{Diff}_{\Sigma}$. The experiments show how many of the differences between two terminologies detected by CEX can be extracted from a straightforward comparison of class hierarchies.

To facilitate the experiments, we use an empty terminology and an SM-05 fragment containing about 140000 axioms. For every number between 10 and 270 with the step of 10 , we generated 500 samples of a random signature containing this number of concepts and 20 roles. The results of the experiments are given in Figure 4. 4(a) shows that, for these signatures, the number of concept names CEX outputs is about five times larger than the number of concept names occurring in differences between the class hierarchies. In 4(b), we do not count the number of differences but analyse how often the two approaches detect differences at all. More precisely, we give the percentage of cases when CEX detects a difference between the two terminologies and when a difference is visible in the class hierarchies. For signatures larger than 200, both approaches almost always detect differences. But for smaller signatures there is again a significant gap between the two approaches.

Scalability. We demonstrated in the previous section that CEX is capable of finding the logical difference in two unmodified versions of SNOMED CT. In order to 
see how CEX's performance scales, we now test it on randomly generated acyclic terminologies of various sizes. Each randomly generated terminology contains a certain number of defined- and primitive concept names and role names. The ratio between concept equations and concept inclusions is fixed, as is the ratio between existential restrictions and conjunctions. The random terminologies were generated for a varying number of defined concept names using the parameters of SM-05: 62 role names; the average number of conjuncts is 2.59; the equality-inclusion ratio is 0.102 ; and the exists-conjunction ratio is 0.652 . For every chosen size, we generate a number of samples consisting of two random terminologies as described above. We apply CEX to find the logical difference of the two terminologies over their joint signature. Figure 5 shows the time and memory consumption of CEX on randomly generated terminologies of various sizes. In 5(a) the maximum length of conjunctions was fixed as two $(\mathrm{M}=2)$, and in 5 (b) the number of conjuncts in each conjunction is randomly selected between two and M. It can be seen that the performance of CEX crucially depends on the length of conjunctions. In 5(b), the curves break off at the point where CEX runs out of memory. For instance, in the case $\mathrm{M}=22$, this happens for terminologies with more than 9500 defined concept names.

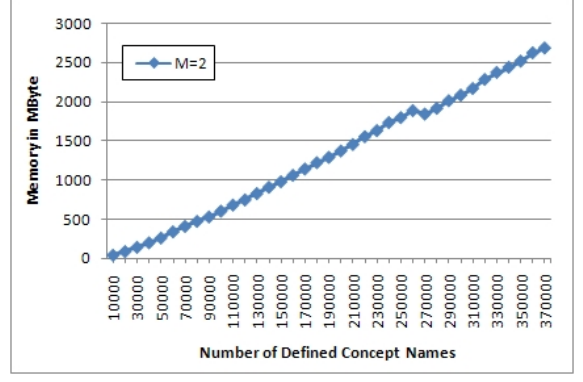

(a) Short conjunctions

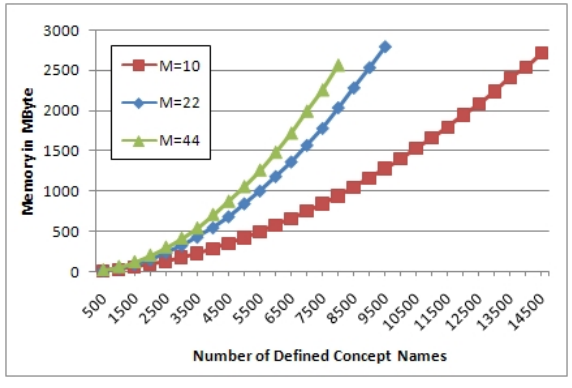

(b) Long conjunctions

Fig. 5. Memory consumption of CEX on randomly generated terminologies

\section{Uniform Interpolation}

Let $\mathcal{T}$ be a terminology and $\Sigma$ a signature. A general TBox $\mathcal{T}_{\Sigma}$ is called a uniform interpolant for $\mathcal{T}$ w.r.t. $\Sigma$ if $\operatorname{sig}\left(\mathcal{T}_{\Sigma}\right) \subseteq \Sigma$ and $\mathcal{T}_{\Sigma}$ and $\mathcal{T}$ are $\Sigma$-inseparable. The question whether uniform interpolants exist for every terminology $\mathcal{T}^{4}$ and signature $\Sigma$ in a logic (i.e., whether the logic has uniform interpolation), has been investigated extensively in the literature, in particular in modal and intuitionistic logic $[15,18,8]$. For instance, modal logic $\mathrm{K}$ has uniform interpolation [18], but S4 does not [8]. Observe that, if a uniform interpolant $\mathcal{T}_{\Sigma}^{\prime}$ of $\mathcal{T}^{\prime}$ w.r.t. $\Sigma$

\footnotetext{
${ }^{4}$ In modal or intuitionistic $\operatorname{logic} \mathcal{T}$ is, of course, a formula.
} 
exists, then $\mathcal{T} \Sigma$-entails $\mathcal{T}^{\prime}$ if, and only if, $\mathcal{T} \models \mathcal{T}_{\Sigma}^{\prime}$. Thus, the problem of deciding $\Sigma$-entailment is reduced to computing a uniform interpolant and standard deduction. Unfortunately, even for $\mathcal{E} \mathcal{L}$-terminologies uniform interpolants do not always exist.

Lemma 6. There exists a $\mathcal{E} \mathcal{L}$-terminology $\mathcal{T}$ and a signature $\Sigma$ such that there does not exist a uniform interpolant of $\mathcal{T}$ w.r.t. $\Sigma$.

Proof. Let $\mathcal{T}=\left\{A_{0} \sqsubseteq B, B \sqsubseteq A_{1} \sqcap \exists r . B\right\}$ and $\Sigma=\left\{A_{0}, A_{1}, r\right\}$. Then a uniform interpolant $\mathcal{T}_{\Sigma}$ would have to axiomatise (using symbols from $\Sigma$ only) the class of interpretations $\mathcal{I}$ satisfying the following condition: if $d_{0} \in A_{0}^{\mathcal{I}}$, then there exists a sequence $d_{0} r^{\mathcal{I}} d_{1} r^{\mathcal{I}} d_{2} r^{\mathcal{I}} \ldots$ with $d_{i} \in A_{1}^{\mathcal{I}}$ for all $i \geq 0$. It is not difficult to show that no such $\mathcal{T}_{\Sigma}$ exists (even in first-order logic).

On the other hand, uniform interpolants always exist for acyclic $\mathcal{E} \mathcal{L}$-terminologies, but minimal uniform interpolants might contain exponentially many axioms.

Theorem 4. Let $\mathcal{T}$ be an acyclic terminology and $\Sigma$ a signature. Then there exists a uniform interpolant of $\mathcal{T}$ w.r.t. $\Sigma$. In the worst case, minimal uniform interpolants have exponentially many axioms.

Proof. First, one can show that $\mathcal{T}_{\Sigma}=\mathcal{T}_{\Sigma}^{l} \cup \mathcal{T}_{\Sigma}^{r}$ is a uniform interpolant for $\mathcal{T}$ w.r.t. $\Sigma$ if $\operatorname{sig}\left(\mathcal{T}_{\Sigma}\right) \subseteq \Sigma$ and

(a) $\mathcal{T} \models C \sqsubseteq A$ if, and only if, $\mathcal{T}_{\Sigma}^{l}=C \sqsubseteq A$, for all $\Sigma$-concepts $C$ and $A \in \Sigma$;

(b) $\mathcal{T}=A \sqsubseteq D$ if, and only if, $\mathcal{T}_{\Sigma}^{r} \models A \sqsubseteq D$, for all $\Sigma$-concepts $D$ and $A \in \Sigma$.

Due to space constraints we cannot describe the construction of $\mathcal{T}_{\Sigma}^{l}$ and $\mathcal{T}_{\Sigma}^{r}$ here, and refer the reader to the Appendix. The following example shows that, in the worst case, minimal uniform interpolants require exponentially many axioms. Let

$$
\mathcal{T}=\left\{A \equiv B_{1} \sqcap \cdots \sqcap B_{n}\right\} \cup\left\{A_{i j} \sqsubseteq B_{i} \mid 1 \leq i, j \leq n\right\} .
$$

and $\Sigma=\{A\} \cup\left\{A_{i j} \mid 1 \leq i, j \leq n\right\}$. Then

$$
\mathcal{T}_{\Sigma}=\left\{A_{1 j_{1}} \sqcap \cdots \sqcap A_{n, j_{n}} \sqsubseteq A \mid 1 \leq j_{1}, \ldots, j_{n} \leq n\right\}
$$

is a uniform interpolant. It is easy to see that no uniform interpolant with less axioms exists. This example shows as well that one has to allow for general TBoxes when constructing uniform interpolants.

The results above show that, at least from a theoretical viewpoint, deciding $\Sigma$ entailment via uniform interpolants is less efficient than the approach discussed before. Still, uniform interpolants are useful for a number of applications, and it would be of interest to see whether this approach is viable for real-world terminologies. 


\section{Discussion}

We have shown that computing the logical difference is tractable for $\mathcal{E} \mathcal{L}$-terminologies and that this approach exhibits differences which are not visible in the class hierarchy. Our experiments with SNOMED CT show that the algorithm can be implemented in such a way that very large terminologies can be compared efficiently.

The following result shows that there is no straightforward way of extending these results to (even acyclic) terminologies in the basic Boolean description logic $\mathcal{A L C}$ (in which concepts can be constructed using, in addition, negation).

Theorem 5. (1) $\Sigma$-entailment is NEXPTIME-hard for acyclic $\mathcal{A L C}$-terminologies.

(2) Uniform interpolants do not always exist for acyclic $\mathcal{A} \mathcal{L C}$-terminologies.

Proof. Point (1) is proved by a reduction of a NExPTIME-hard problem for conservative extensions in modal logic [7],

Consider the class $\mathcal{K}$ of interpretations $\mathcal{I}=\left(\Delta^{\mathcal{I}}, r^{\mathcal{I}}, A_{1}^{\mathcal{I}}, \ldots\right)$ in which $\left(\Delta^{\mathcal{I}}, r^{\mathcal{I}}\right)$ is a tree of depth 1 ; i.e., there exists $d_{\mathcal{I}} \in \Delta^{\mathcal{I}}$ such that

$$
r^{\mathcal{I}}=\left\{\left(d_{\mathcal{I}}, d\right) \mid d \in \Delta^{\mathcal{I}} \backslash\left\{d_{\mathcal{I}}\right\}\right\}
$$

and $r^{\mathcal{I}} \neq \emptyset$. A $\mathcal{A L C}_{r}$ concept is an $\mathcal{A L C}$ concept whose sole role name is $r$. Call a $\mathcal{A L C}_{r}$ concept $C \sqcap D$ a $\mathcal{K}$-conservative extension of $C$ if, for every $\mathcal{A L C}$-concept $E$ with $\operatorname{sig}(E) \subseteq \operatorname{sig}(C)$ the following holds: if $C \sqcap E$ is satisfiable in $d_{\mathcal{I}}$ in an interpretation $\mathcal{I} \in \mathcal{K}$, then $C \sqcap D \sqcap E$ is satisfiable in $d_{\mathcal{I}^{\prime}}$ in an interpretation $\mathcal{I}^{\prime} \in \mathcal{K}$. It is proved in [?] (in the language of modal logic) that it is NExpTimehard to decide whether $C \sqcap D$ is a $\mathcal{K}$-conservative extension of $C$. We now prove that the following two conditions are equivalent, for all $\mathcal{A L C}_{r}$-concepts $C$ and $D$ :

- $C \sqcap D$ is a $\mathcal{K}$-conservative extension of $C$;

- $\mathcal{T} \Sigma$-entails $\mathcal{T}^{\prime}$, where $\mathcal{T}=\{A \sqsubseteq C \sqcap \exists r . \top \sqcap \forall r . \forall r . \perp \sqcap X\}, \mathcal{T}^{\prime}=\mathcal{T} \cup\{X \sqsubseteq D\}$ ( $A$ and $X$ are fresh concept names), and $\Sigma=\operatorname{sig}(C) \cup\{A\}$.

Suppose $C \sqcap D$ is not a $\mathcal{K}$-conservative extension of $C$. Take $E$ with $\operatorname{sig}(E) \subseteq$ $\operatorname{sig}(C)$ such that $C \sqcap E$ is satisfied in $d_{\mathcal{I}}$ in a $\mathcal{K}$-interpretation $\mathcal{I}$ but $C \sqcap D \sqcap E$ is not satisfiable in such an interpretation. Then $\mathcal{T}^{\prime} \models A \sqsubseteq \neg E$ but $\mathcal{T} \forall A \sqsubseteq \neg E$.

Conversely, assume that $C \sqcap D$ is a $\mathcal{K}$-conservative extension of $C$. Assume that $\mathcal{T}^{\prime} \models E \equiv \top$ but $\mathcal{T} \not \models E \equiv \top$ for some $E$ with $\operatorname{sig}(E) \subseteq \operatorname{sig}(C) \cup\{A\}$. Take an interpretation $\mathcal{I}$ satisfying $\mathcal{T}$ with $(\neg E)^{\mathcal{I}} \neq \emptyset$. We may assume that $\left(\Delta^{\mathcal{I}}, r^{\mathcal{I}}\right)$ is a tree with $d_{0} \notin E^{\mathcal{I}}$ for its root $d_{0}$ such that each node has either no $r^{\mathcal{I}}$ successor or infinitely many $r^{\mathcal{I}}$ successors.

If $A^{\mathcal{I}}=\emptyset$, then we obtain an interpretation satisfying $\mathcal{T}^{\prime}$ and in which $\neg E$ is satisfied by assuming $X^{\mathcal{I}}=\emptyset$. Thus, we have derived a contradiction.

Assume now that $A^{\mathcal{I}} \neq \emptyset$. Then $d_{0}$ has $r^{\mathcal{I}}$-successors. As $C \sqcap D$ is a $\mathcal{K}$ conservative extension of $C$ and using $A^{\mathcal{I}} \subseteq(\exists r . \top \sqcap \forall r . \forall r . \perp)^{\mathcal{I}}$, we can change the interpretation $\mathcal{I}$ to an interpretation $\mathcal{I}^{\prime}$ by keeping the interpretation of $r$ and $A$ fixed, setting $X^{\mathcal{I}}=A^{\mathcal{I}}$, and changing the interpretation of the remaining concept names $B$ in such a way that 
- for all $d \in \Delta^{\mathcal{I}}$ such that $d \notin A^{\mathcal{I}} \cup\left\{d^{\prime} \mid d \in A^{\mathcal{I}},\left(d, d^{\prime}\right) \in r^{\mathcal{I}}\right\}, d \in B^{\mathcal{I}}$ iff $d \in B^{\mathcal{I}^{\prime}}$

$-A^{\mathcal{I}^{\prime}} \subseteq(C \sqcap D)^{\mathcal{I}^{\prime}}$

- for all subconcepts $F$ of $E$ and $d \in A^{\mathcal{I}}, d \in F^{\mathcal{I}}$ iff $d \in F^{\mathcal{I}^{\prime}}$.

Then $\mathcal{I}^{\prime}$ satisfies $\mathcal{T}^{\prime}$ and $d_{0} \in(\neg E)^{\mathcal{I}^{\prime}}$. Again we have derived a contradiction.

Point (2). We rewrite the terminology from Lemma 6 . Let $\mathcal{T}=\left\{A \sqsubseteq\left(\neg A_{0} \sqcup\right.\right.$ B) $\left.\sqcap\left(\neg B \sqcup\left(A_{1} \sqcap \exists r . B\right)\right)\right\}$ and $\Sigma=\left\{A, A_{0}, A_{1}, r\right\}$. It follows from the proof of Lemma 6 that there does not exist a general $\mathcal{A L C}$-TBox $\mathcal{T}_{\Sigma}^{A}$ axiomatising (using only the symbols from $\Sigma$ ) the class $\mathcal{S}$ of interpretations $\mathcal{I}$ satisfying the following conditions: $A^{\mathcal{I}}=\Delta^{\mathcal{I}}$ and if $d_{0} \in A_{0}^{\mathcal{I}}$, then there exists a sequence $d_{0} r^{\mathcal{I}} d_{1} r^{\mathcal{I}} d_{2} r^{\mathcal{I}} \ldots$ with $d_{i} \in A_{1}^{\mathcal{I}}$ for all $i \geq 0$. Now assume that there exists a uniform interpolant $\mathcal{T}_{\Sigma}$ of $\mathcal{T}$ w.r.t. $\Sigma$. Then $\mathcal{T}_{\Sigma} \cup\{A \equiv \top\}$ would be an axiomatisation of $\mathcal{S}$ and we have derived a contradiction.

Point (2) of Theorem 5 is slightly unexpected, because it shows that it is not possible to lift results from modal logic $\mathrm{K}$ (which has uniform interpolation) to acyclic $\mathcal{A L C}$-terminologies. Besides of considering extensions of our approach to languages with additional concept constructors, such as $\mathcal{A L C}$, directions for future research include terminologies with additional role boxes. SNOMED CT has an additional role box consisting of implications $r \sqsubseteq r^{\prime}, r \circ s \sqsubseteq r$ (rightidentities), and $s \circ r \sqsubseteq r$ (left-identities), where $r, s, r^{\prime}$ are role names. It is not difficult to extend the algorithm (and implementation) presented in this paper to terminologies containing implications of the first type, but it remains open whether $\Sigma$-entailment is still tractable for additional role boxes containing leftand right-identities.

Finally, for the system CEX to be useful in practice, the outputs Diff $\mathrm{L}_{\Sigma}$ and DiffR $_{\Sigma}$ have to be expanded by suggesting, for $A \in \operatorname{DiffR}_{\Sigma}, \Sigma$-concepts $C$ such that $C \sqsubseteq A \in \operatorname{Diff}_{\Sigma}$, and similarly for DiffL $L_{\Sigma}$. Computing such $C$ 's is straightforward by unfolding the concept $\xi_{A}$ relative to Noimply ${ }_{\mathcal{T}, \Sigma}$. However, even this might not provide enough information, because for the user it could be difficult to find out which difference between the axioms of the two terminologies has caused a certain $\Sigma$-difference. Thus, as a second step one might consider pinpointing algorithms explaining from which axioms of a terminology a counterexample $C \sqsubseteq A$ is derivable [3].

\section{References}

1. F. Baader. Terminological cycles in a description logic with existential restrictions. In Proceedings of IJCAI'03, pp. 325-330. Morgan Kaufmann, 2003. Long version available as LTCS Report 02-02.

2. F. Baader, C. Lutz, and B. Suntisrivaraporn. CEL - a polynomial-time reasoner for life science ontologies. In Proceedings of IJCAR'06, vol. 4130 of LNAI, pp. 287-291. Springer-Verlag, 2006.

3. F. Baader, R. Peñaloza, and B. Suntisrivaraporn. Pinpointing in the description logic $\mathcal{E L}^{+}$. In Proceedings of KI'07, vol. 4667 of LNAI, pp. 52-67. Springer, 2007.

4. The Caml team. http://caml.inria.fr/contact.en.html. 
5. A. Flögel, H. K. Büning, and T. Lettmann. On the restricted equivalence of subclasses of propositional logic. ITA, 27(4):327-340, 1993.

6. S. Ghilardi, C. Lutz, and F. Wolter. Did I damage my ontology? a case for conservative extensions in description logics. In Proceedings of KR'06, pp. 187-197. AAAI Press, 2006.

7. S. Ghilardi, C. Lutz, F. Wolter, and M. Zakharyaschev. Conservative extensions in modal logics. In Proceedings of AiML-6, pp. 187-207. College Publications, 2006.

8. S. Ghilardi and M. Zawadowski. Undefinability of propositional quantifiers in the modal system S4. Studia Logica, 55(2):259-271, 1995.

9. B. C. Grau, I. Horrocks, Y. Kazakov, and U. Sattler. Just the right amount: extracting modules from ontologies. In Proceedings of $W W W^{\prime} 0 \%$, pp. 717-726. ACM, 2007.

10. M. Hofmann. Proof-theoretic approach to description logic. In Proceedings of LICS'05, pp. 229-237. IEEE Computer Society, 2005.

11. B. Konev, D. Walther, and F. Wolter. The logical difference problem for description logic terminologies. http://www.csc.liv.ac.uk/ frank/publ/publ.html, manuscript, 2008.

12. C. Lutz, D. Walther, and F. Wolter. Conservative extensions in expressive description logics. In Proceedings of IJCAI'07, pp. 453-458. AAAI Press, 2007.

13. C. Lutz and F. Wolter. Conservative extensions in the lightweight description logic $\mathcal{E} \mathcal{L}$. In Proceedings of $C A D E^{\prime} 0 \%$, vol. 4603 of $L N C S$, pp. 84-99. Springer, 2007.

14. N. F. Noy and M. Musen. Promptdiff: A fixed-point algorithm for comparing ontology versions. In Proceedings of AAAI'02, pp. 744-750. AAAI Press, 2002.

15. A. Pitts. On an interpretation of second-order quantification in first-order intuitionistic propositional logic. Journal of Symbolic Logic, 57(1):33-52, 1992.

16. V. Sofronie-Stokkermans. Interpolation in local theory extensions. In IJCAR'06, pp. 235-250, 2006.

17. K. Spackman. Managing clinical terminology hierarchies using algorithmic calculation of subsumption: Experience with SNOMED-RT. JAMIA, 2000. Fall Symposium Special Issue.

18. A. Visser. Uniform interpolation and layered bisimulation. In Gödel'96 (Brno, 1996), vol. 6 of Lecture Notes Logic, pp. 139-164. Springer, 1996. 


\section{A Proof of Lemma 3}

Lemma 3. For every terminology $\mathcal{T}$, one can construct in polynomial time a normalised terminology $\mathcal{T}^{\prime}$ of polynomial size in $|\mathcal{T}|$ such that $\operatorname{sig}(\mathcal{T}) \subseteq \operatorname{sig}\left(\mathcal{T}^{\prime}\right)$, $\mathcal{T}^{\prime}=\mathcal{T}$, and for every model $\mathcal{I}$ of $\mathcal{T}$, there exists a model $\mathcal{J}$ of $\mathcal{T}^{\prime}$ which coincides with $\mathcal{I}$ on $\Sigma$. Moreover, $\mathcal{T}^{\prime}$ is acyclic if $\mathcal{T}$ is acyclic.

Proof. Given a terminology $\mathcal{T}$, construct a normalised terminology $\mathcal{T}^{\prime}$ in four steps as follows: First, replace $C$ in each occurrence of $\exists r . C$, where $C$ is not a concept name, with a fresh concept name $A$ and add the concept definition $A \equiv C$ to the terminology. Repeat this step exhaustively.

Second, replace every $\exists r_{i} . B_{i}$ in each axiom with a right-hand side of the form $F \sqcap \exists r_{1} . B_{1} \sqcap \ldots \sqcap \exists r_{m} . B_{m}$, where $F$ is a conjunction of concept names, with a fresh concept name $B_{i}^{\prime}$ and add the concept definition $B_{i}^{\prime} \equiv \exists r_{i} . B_{i}$ to the terminology.

Third, consider any concept name $A$ such that there are sequences $B_{0}, \ldots, B_{n-1}$ and $F_{0}, \ldots, F_{n}$, where the $F_{i}$ are conjunctions of concept names, such that the terminology contains the concept definitions $A \equiv F_{0}$ and $B_{i} \equiv F_{i+1}$, for $i<n$, where $B_{i}$ is a conjunct of $F_{i}$ and $A$ a conjunct of $F_{n}$. Let $F_{n}^{\prime}$ be the conjunction of concept names in $F_{n}$ except $A$. Let, recursively, $F_{i-1}^{\prime}$ be the result of replacing the conjunct $B_{i-1}$ in $F_{i-1}$ with the conjunction $F_{i}^{\prime}$, for $1 \leq i \leq n$. Replace the concept definition $A \equiv F_{0}$ in the terminology with the primitive concept definition $A \sqsubseteq F_{0}^{\prime}$.

Fourth, for each axiom $A \equiv F$ or $A \sqsubseteq F$, where $F$ is a conjunction of concept names, replace every conjunct $B$ in $F$ for which there is a $B \equiv F^{\prime}$ in the terminology, where $F^{\prime}$ is a conjunction of non-conjunctive concept names, with $F^{\prime}$.

To see that the construction indeed yields a normalised terminology $\mathcal{T}^{\prime}$, observe that steps 1 and 2 ensure that each axiom has one of the following forms: $A \equiv \exists r . B$ or $A \sqsubseteq \exists r . B$, where $B$ is a concept name, or $A \equiv F$ or $A \sqsubseteq F$ with $F$ being a conjunction of (possibly conjunctive) concept names. Step 3 breaks cycles in concept definitions and Step 4 takes care that all conjuncts of the conjunction of concept names $F$ in the right-hand side of each axiom of the form $A \equiv F$ or $A \sqsubseteq F$ are non-conjunctive concept names. It is readily verified that $\mathcal{T}^{\prime}$ is acyclic if $\mathcal{T}$ is acyclic as none of the above steps introduces cycles in concept definitions.

We now show that $\mathcal{T}^{\prime}$ can be obtained in polynomial time and that $\mathcal{T}^{\prime}$ is of polynomial size in $|\mathcal{T}|$. Let $n$ be the number of axioms in $\mathcal{T}$ and $c$ the maximal length of an axiom's right-hand side in $\mathcal{T}$. Clearly, step 1 and 2 each introduce not more than $c \cdot n$ many new axioms, increasing the total number of axioms to at most $3 n c$. Steps 3 and 4 do not increase the number of axioms, but the length of their right-hand sides. The length of the right-hand side of an axiom can increase to at most the sum of the lengths of the right-hand sides of all axioms, i.e., $3 n c^{2}$ is an upper bound for each right-hand side. The upper bound for the running time of the construction is $9 n^{2} c^{3}$. Hence, the size of $\mathcal{T}^{\prime}$ and the running time of the construction are both in $\mathcal{O}(n)$. 
Notice that every new concept name occurs on the left-hand side of a unique concept definition $A \equiv C$ in $\mathcal{T}^{\prime}$. Thus, every model $\mathcal{I}$ of $\mathcal{T}$ can be expanded to a model $\mathcal{J}$ of $\mathcal{T}^{\prime}$ by interpreting the fresh concept names in $\operatorname{sig}\left(\mathcal{T}^{\prime}\right) \backslash \operatorname{sig}(\mathcal{T})$ by setting $A^{\mathcal{J}}=C^{\mathcal{I}}$. Clearly, $\left.\mathcal{I}\right|_{\Sigma}=\left.\mathcal{J}\right|_{\Sigma}$, for all $\Sigma \subseteq \operatorname{sig}(\mathcal{T})$.

Moreover, it is readily checked that $\mathcal{T}^{\prime} \models \mathcal{T}$.

\section{B Proof of Lemma 5}

We prove a slightly stronger version:

Theorem 6. Let $\mathcal{T}^{\prime}$ be a terminology such that $\operatorname{sig}\left(\mathcal{T}^{\prime}\right) \cap \Sigma^{\text {fresh }}=\emptyset$. Then the following conditions are equivalent, for all concept names $A, E \in \operatorname{sig}(\mathcal{T}) \cup \Sigma$ :

1. $\mathcal{T}^{\prime} \cup$ Noimply $_{\mathcal{T}, \Sigma} \models \xi \sqsubseteq E$, for some $\xi \in \operatorname{noimply}_{\mathcal{T}, \Sigma}(A)$;

2. $\mathcal{T}^{\prime} \models C \sqsubseteq E$, for some $n \geq 0$ and $C \in$ noimply $_{\mathcal{T}, \Sigma}^{n}(A)$.

The proof of (2) implies (1) is clear. So we concentrate on the direction from (1) to (2). The proof consists of two lemmata. Assume $\mathcal{T}$ and $\Sigma$ are given. In Figure 6 , we define sets wimply $\mathcal{T}, \Sigma^{n}(A), n \geq 0$, of concept names, and terminologies Noimply ${ }_{\mathcal{T}, \Sigma}^{n}(A)$.

Lemma 7. Let $\Sigma_{0}=\Sigma^{\text {fresh }} \cup \Sigma^{\text {freshm }}$ and $A \in \Sigma \cup \operatorname{Sig}(\mathcal{T})$ non-conjunctive in $\mathcal{T}$.

- For every interpretation $\mathcal{I}_{1}$ satisfying $\bigcup_{n \geq 0} \operatorname{Noimply}_{\mathcal{T}, \Sigma}^{n}(A) \cup\left\{\alpha^{n}\right\}$ with $d \in$ $\left(s_{A}^{0}\right)^{\mathcal{I}_{1}}$ there exists an interpretation $\mathcal{I}_{2}$ which coincides with $\mathcal{I}_{1}$ on $\left(\mathrm{N}_{1} \cup\right.$ $\left.\mathrm{N}_{\mathrm{R}}\right) \backslash \Sigma_{0}$ and satisfies Noimply $_{\mathcal{T}, \Sigma}$ such that $d \in \xi_{A}^{\mathcal{I}_{2}}$.

- Conversely, for every interpretation $\mathcal{I}_{1}$ satisfying Noimply $_{\mathcal{T}, \Sigma}$ with $d \in \xi_{A}^{\mathcal{I}_{1}}$ there exists an interpretation $\mathcal{I}_{2}$ which coincides with $\mathcal{I}_{1}$ on $\left(\mathrm{N}_{1} \cup \mathrm{N}_{\mathrm{R}}\right) \backslash \Sigma_{0}$ and satisfies $\bigcup_{n>0}$ Noimply $_{\mathcal{T}, \Sigma}^{n}(A) \cup\left\{\alpha^{n}\right\}$ such that $d \in\left(s_{A}^{0}\right)^{\mathcal{I}_{2}}$.

In particular, for every terminology $\mathcal{T}^{\prime}$ with $\operatorname{sig}\left(\mathcal{T}^{\prime}\right) \cap \Sigma_{0}=\emptyset$ and $A, E \in \Sigma \cup$ $\operatorname{sig}(\mathcal{T})$, the following are equivalent:

$-\mathcal{T}^{\prime} \cup$ Noimply $_{\mathcal{T}, \Sigma} \models \xi \sqsubseteq E$, for some $\xi \in$ noimply $_{\mathcal{T}, \Sigma}(A)$;

- there exists $n \geq 0$ such that $\mathcal{T}^{\prime} \cup \bigcup_{n \geq i \geq 0} \operatorname{Noimply}_{\mathcal{T}, \Sigma}^{i}(A) \cup\left\{\alpha^{i}\right\} \models B \sqsubseteq E$, for some $B \in$ wimply $_{\mathcal{T}, \Sigma}^{0}(A)$.

Proof. Suppose $\mathcal{I}_{1}$ satisfies $\bigcup_{n \geq 0}$ Noimply $_{\mathcal{T}, \Sigma}^{n}(A) \cup\left\{\alpha_{n}\right\}$ and $d \in\left(s_{A}^{0}\right)^{\mathcal{I}_{1}}$. Define an interpretation $\mathcal{I}_{2}$ by interpreting the concepts names $\xi_{B}, B$ non-conjunctive in $\mathcal{T}$ as follows: if $A \prec_{\mathcal{T}}^{*} B$ or $A=B$, then

$$
\xi_{B}^{\mathcal{I}_{2}}=\bigcup_{n \geq 0}\left(s_{B}^{n}\right)^{\mathcal{I}_{1}}
$$

and $\xi_{B}^{\mathcal{I}_{2}}=\emptyset$, otherwise. Also set

$$
\mathrm{All}_{\Sigma}^{\mathcal{I}_{2}}=\bigcup_{n \geq 0}\left(\mathrm{All} \Sigma_{\Sigma}^{n}\right)^{\mathcal{I}_{1}}
$$


Input: normalised terminology $\mathcal{T}$ and signature $\Sigma$.

Output: Sets wimply ${ }_{\mathcal{T}, \Sigma}^{n}(A), n \geq 0$, of concept names, and terminologies Noimply ${ }_{\mathcal{T}, \Sigma}^{n}(A), n \geq 0$, where $A$ is a concept name.

Let $\Sigma^{\text {freshm }}$ denote a set of fresh concept names consisting of AlI ${ }_{\Sigma}^{n}, n \geq 0$, and $s_{A}^{n}$, $n \geq 0$, for $A$ non-conjunctive in $\mathcal{T}$. Let $\alpha^{n}, n \geq 0$, be the CI

$$
\mathrm{All} I_{\Sigma}^{n} \sqsubseteq \prod_{r \in \Sigma} \exists r \cdot\left(\prod_{A^{\prime} \in \Sigma} A^{\prime} \sqcap \mathrm{A} \|_{\Sigma}^{n+1}\right)
$$

and define, for each $A$, sets wimply ${ }_{\mathcal{T}, \Sigma}^{n}(A)$ and terminologies Noimply $\operatorname{No}_{\mathcal{T}, \Sigma}^{n}(A)$ inductively as follows.

- If $A$ is pseudo-primitive in $\mathcal{T}$, then

$$
\text { wimply }_{\mathcal{T}, \Sigma}^{n}(A)=\left\{s_{A}^{n}\right\}, \quad \operatorname{Noimply}_{\mathcal{T}, \Sigma}^{n}(A)=\left\{s_{A}^{n} \sqsubseteq \prod_{A^{\prime} \in\left(\Sigma \backslash \operatorname{pre}_{\mathcal{T}}^{\Sigma}(A)\right)} A^{\prime} \sqcap \mathrm{All}_{\Sigma}^{0}\right\} ;
$$

- If $A$ is conjunctive in $\mathcal{T}$ and $A \equiv F \in \mathcal{T}$, then

$$
\text { wimply }_{\mathcal{T}, \Sigma}^{n}(A)=\bigcup_{B \in F} \text { wimply }_{\mathcal{T}, \Sigma}^{n}(B), \quad \operatorname{Noimply}_{\mathcal{T}, \Sigma}^{n}(A)=\bigcup_{B \in F} \operatorname{Noimply}_{\mathcal{T}, \Sigma}^{n}(B)
$$

- If $A \equiv \exists r . B \in \mathcal{T}$, then wimply ${ }_{\mathcal{T}, \Sigma}^{n}(A)=\left\{s_{A}^{n}\right\}$ and Noimply $\operatorname{No}_{\mathcal{T}, \Sigma}^{n}(A)=\left\{\beta_{n}\right\}$ (for $n=0)$, Noimply $\operatorname{T}_{\mathcal{T}, \Sigma}^{n}(A)=\left\{\beta_{n}\right\} \cup \operatorname{Noimply}_{\mathcal{T}, \Sigma}^{n-1}(B)$ (for $\left.n>0\right)$, where

$$
\beta_{n}=s_{A}^{n} \sqsubseteq\left(\prod_{A^{\prime} \in\left(\Sigma \backslash \operatorname{pre}_{\mathcal{T}}^{\Sigma}(A)\right)} A^{\prime}\right) \sqcap\left(\prod_{r \neq s \in \Sigma} \exists s .\left(\prod_{A^{\prime} \in \Sigma} A^{\prime} \sqcap \mathrm{All}_{\Sigma}^{0}\right)\right) \sqcap \prod_{E \in \operatorname{wimply}_{\mathcal{T}, \Sigma}^{n+1}(B)} \exists r . E .
$$

Fig. 6. Computing Noimply ${ }_{\mathcal{T}, \Sigma}^{n}(A)$ and wimply $_{\mathcal{T}, \Sigma}^{n}(A)$

It is not difficult to show that $\mathcal{I}_{2}$ is as required. Conversely, suppose $\mathcal{I}_{1}$ satisfies Noimply $_{\mathcal{T}, \Sigma}$ and $d \in \xi_{A}^{\mathcal{I}_{1}}$. Define $\mathcal{I}_{2}$ by

$$
\begin{gathered}
\left(s_{B}^{n}\right)^{\mathcal{I}_{2}}=\xi_{B}^{\mathcal{I}_{1}} \\
\left(\mathrm{~A} \|_{\Sigma}^{n}\right)^{\mathcal{I}_{2}}=\mathrm{All}_{\Sigma}^{\mathcal{I}_{1}},
\end{gathered}
$$

for all $n \geq 0$. Again, it is not difficult to see that $\mathcal{I}_{2}$ is as required.

The second part follows using compactness.

The following lemma follows immediately from the definitions:

Lemma 8. Let $\Sigma_{0}=\Sigma^{\text {fresh }} \cup \Sigma^{\text {freshm }}, \mathcal{T}^{\prime}$ a terminology with $\operatorname{sig}\left(\mathcal{T}^{\prime}\right) \cap \Sigma_{0}=\emptyset$, and $A, E \in \Sigma \cup \operatorname{sig}(\mathcal{T})$. The following are equivalent:

- there exists $n \geq 0$ such that $\mathcal{T}^{\prime} \models C \sqsubseteq E$, for some $C \in \operatorname{noimply}_{\mathcal{T}, \Sigma}^{n}(A)$.

- there exists $n \geq 0$ such that $\mathcal{T}^{\prime} \cup \bigcup_{n \geq i \geq 0} \operatorname{Noimply}_{\mathcal{T}, \Sigma}^{i}(A) \cup\left\{\alpha^{i}\right\} \models B \sqsubseteq E$, for some $B \in$ wimply $_{\mathcal{T}, \Sigma}^{0}(A)$. 
Input: normalised acyclic terminologies $\mathcal{T}$ and $\mathcal{T}^{\prime}$ and signature $\Sigma$ with $\Sigma \subseteq \operatorname{sig}\left(\mathcal{T}^{\prime}\right) \subseteq$ $\operatorname{sig}(\mathcal{T})$.

Output: Sets $O_{A^{\prime}}$, one for every concept name $A^{\prime} \in \operatorname{sig}(\mathcal{T})$.

The algorithm uses the following set

$O=\left\{\left(A^{\prime}, A\right) \in \operatorname{sig}(\mathcal{T}) \times \operatorname{sig}(\mathcal{T}) \mid \forall B \in \Sigma:\right.$ if $\mathcal{T}^{\prime} \models A^{\prime} \sqsubseteq B$ then $\left.\mathcal{T} \models A \sqsubseteq B\right\}$.

- If $A^{\prime}$ is primitive in $\mathcal{T}^{\prime}$, or $A \equiv \exists s . A^{\prime} \in \mathcal{T}^{\prime}$ for an $s \notin \Sigma$, or $A \sqsubseteq \exists s . A^{\prime} \in \mathcal{T}^{\prime}$ for an $s \notin \Sigma$, then

$$
O_{A^{\prime}}=\left\{A \mid\left(A^{\prime}, A\right) \in O\right\} .
$$

- If $A^{\prime} \equiv A_{1}^{\prime} \sqcap \ldots \sqcap A_{k}^{\prime}$ or $A^{\prime} \sqsubseteq A_{1}^{\prime} \sqcap \ldots \sqcap A_{k}^{\prime}$ in $\mathcal{T}^{\prime}$, then

$$
O_{A^{\prime}}=\left\{A \mid\left(A^{\prime}, A\right) \in O\right\} \cap O_{A_{1}^{\prime}} \cap O_{A_{2}^{\prime}} \cap \ldots \cap O_{A_{k}^{\prime}} .
$$

- If $A^{\prime} \equiv \exists r . A_{1}^{\prime}$ or $A^{\prime} \sqsubseteq \exists r . A_{1}^{\prime}$ in $\mathcal{T}^{\prime}$, where $r \in \Sigma$, then

$$
O_{A^{\prime}}=\left\{A \mid\left(A^{\prime}, A\right) \in O \text { and there exists } A_{1} \in O_{A_{1}^{\prime}} \text { such that } \mathcal{T} \models A \sqsubseteq \exists r . A_{1}\right\}
$$

Fig. 7. Computing $O_{A^{\prime}}$ for $A^{\prime} \in \operatorname{sig}(\mathcal{T})$

\section{Algorithm computing $\operatorname{DiffL}\left(\mathcal{T}, \mathcal{T}^{\prime}\right)$}

We present an algorithm computing $\operatorname{DiffL}_{\Sigma}\left(\mathcal{T}, \mathcal{T}^{\prime}\right)$ for acyclic terminologies $\mathcal{T}$ and $\mathcal{T}^{\prime}$. For expositional reasons, we assume that $\Sigma \subseteq \operatorname{sig}\left(\mathcal{T}^{\prime}\right) \subseteq \operatorname{sig}(\mathcal{T})$.

Proposition 1. For all $A^{\prime} \in \Sigma, A^{\prime} \in \operatorname{DiffL}_{\Sigma}\left(\mathcal{T}, \mathcal{T}^{\prime}\right)$ iff $A^{\prime} \notin O_{A^{\prime}}$, where the set $O_{A^{\prime}}$ is computed by the algorithm given in Figure 7.

Proof. We show that, for every $A, A^{\prime} \in \operatorname{sig}(\mathcal{T})$, the set $O_{A^{\prime}}$ has the following property:

(*) $A \in O_{A^{\prime}}$ if, and only if, for every $\Sigma$-concept $D$, if $\mathcal{T}^{\prime} \models A^{\prime} \sqsubseteq D$, then $\mathcal{T}=A \sqsubseteq D$.

Suppose that $A, A^{\prime} \in \operatorname{sig}(\mathcal{T})$ are such that for every $\Sigma$-concept $D$, if $\mathcal{T}^{\prime} \models A^{\prime} \sqsubseteq$ $D$, then $\mathcal{T}=A \sqsubseteq D$. It is easy to see that $A \in O_{A^{\prime}}$.

It can readily be verified that the property $(*)$ holds for concept names $A^{\prime}$ covered in Point 1 of Figure 7.

We now show property $(*)$ for $A^{\prime}$ defined as $A^{\prime} \equiv A_{1}^{\prime} \sqcap \ldots \sqcap A_{k}^{\prime}$ or $A^{\prime} \sqsubseteq$ $A_{1}^{\prime} \sqcap \ldots \sqcap A_{k}^{\prime}$ in $\mathcal{T}^{\prime}$. Assume that for $A_{1}^{\prime}, \ldots, A_{k}^{\prime}$ property $(*)$ holds. We show that if $A \in O_{A^{\prime}}$ and $\mathcal{T}^{\prime} \models A^{\prime} \sqsubseteq D$, for some $\Sigma$-concept $D$, then $\mathcal{T} \models A \sqsubseteq D$ by induction on construction of $D$. If $D$ is atomic, then this follows from $\left(A^{\prime}, \bar{A}\right) \in O$. If $D$ is a conjunction, $D_{1} \sqcap \ldots \ldots \sqcap D_{m}$, then $\mathcal{T}^{\prime} \models A^{\prime} \sqsubseteq D$ iff $\mathcal{T}^{\prime} \models A^{\prime} \sqsubseteq D_{i}$ for every $1 \leq i \leq m$. By induction hypothesis, $\mathcal{T} \models A \sqsubseteq D_{i}$ for all $1 \leq i \leq m$ and, hence, $\mathcal{T} \models A \sqsubseteq D$. If $D$ is of the form $\exists r . D_{1}$ then, by Lemma 1 , there exists $i: 1 \leq i \leq k$ such that $\mathcal{T}^{\prime} \models A_{i}^{\prime} \sqsubseteq \exists r . D$. Since for $A_{i}^{\prime}$ the property (*) holds and $A \in O_{A_{i}^{\prime}}$, we have $\mathcal{T} \models A \sqsubseteq \exists$ r.D. 
Finally, we show property (*) for $A^{\prime}$ defined as $A^{\prime} \equiv \exists r . A_{1}^{\prime}$ or $A^{\prime} \sqsubseteq \exists r . A_{1}^{\prime}$ in $\mathcal{T}^{\prime}$, where $r \in \Sigma$. Assume that for $A_{1}^{\prime}$ property $(*)$ holds. We show that if $A \in O_{A^{\prime}}$ and $\mathcal{T}^{\prime} \models A^{\prime} \sqsubseteq D$, for some $\Sigma$-concept $D$, then $\mathcal{T} \models A \sqsubseteq D$ by induction on construction of $D$. The case of $D$ being atomic or a conjunction are similar to the ones considered above. If $D$ is of the form $\exists$ r. $D_{1}$ then, by Lemma $1, \mathcal{T}^{\prime} \models A_{1}^{\prime} \sqsubseteq D_{1}$. Let $A_{1} \in O_{A_{1}^{\prime}}$ be such that $\mathcal{T} \models A \sqsubseteq \exists r$. $A_{1}$. Since for $A_{1}^{\prime}$ the property $(*)$ holds, we have $\mathcal{T} \models A_{1} \sqsubseteq D_{1}$ and so $\mathcal{T} \models \exists r . A_{1} \sqsubseteq \exists r . D_{1}$. Since $\mathcal{T} \models A \sqsubseteq \exists r$. $A_{1}$, we have $\mathcal{T} \models A \sqsubseteq \exists r . D_{1}$.

The algorithm given in Figure 7 finds counterexamples of the form $A \sqsubseteq C$ in $O\left(|\mathcal{T}| \times\left|\mathcal{T}^{\prime}\right| \times|\Sigma|+T+T^{\prime}\right)$ time and requires $O\left(|\mathcal{T}| \times\left|\mathcal{T}^{\prime}\right| \times|\Sigma|\right)$ space, where $T$ and $T^{\prime}$ are the times taken to fully classify $\mathcal{T}$ and $\mathcal{T}^{\prime}$, respectively. In fact, the sets $O_{A^{\prime}}$ represent the largest simulation relation between the canonical models of $\mathcal{T}^{\prime}$ and $\mathcal{T}$. The reduction of the existence of counterexamples to the $\Sigma$-entailment of the form $A \sqsubseteq C$ to the existence of a simulation relation on graphs was given in [12].

\section{Proof of property $(\dagger)$}

(a) Suppose $E$ is pseudo-primitive in $\mathcal{T}^{\prime}$. Now, we consider cases depending on how $A$ is defined in $\mathcal{T}$. If $A$ is pseudo-primitive in $\mathcal{T}$ then, by Theorem 6 , $\mathcal{T}^{\prime} \cup$ Noimply $_{\mathcal{T}, \Sigma} \models \xi \sqsubseteq E$ if, and only if, there exist $n \geq 0$ such that $\mathcal{T}^{\prime} \models$

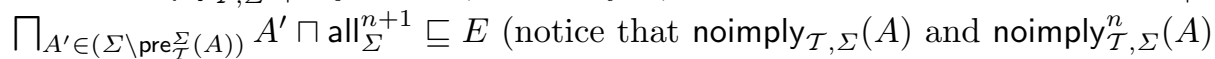
are both singletons in this case). By Lemma 1, this holds if, and only if, $\mathcal{T}^{\prime}=$ $B \sqsubseteq E$ for some $B \in\left(\Sigma \backslash \operatorname{pre}_{\mathcal{T}}^{\Sigma}(A)\right)$. Thus, $\mathcal{T}^{\prime} \cup$ Noimply $_{\mathcal{T}, \Sigma} \not \models \xi \sqsubseteq E$ if, and only if, $\operatorname{pre}_{\mathcal{T}^{\prime}}^{\Sigma}(E) \subseteq \operatorname{pre}_{\mathcal{T}}^{\Sigma}(\xi)$.

The case when $A \equiv \exists r \cdot A^{\prime}$ in $\mathcal{T}$ is similar, and for conjunctive $A$ 's, ( $\dagger$ ) follows by induction.

(b) The case of $E \equiv E_{1} \sqcap \ldots \sqcap E_{k}$ is easy and left to the reader.

(c) Assume now that $E \equiv \exists r . E^{\prime} \in \mathcal{T}^{\prime}$.

If $r \notin \Sigma$ or $\mathcal{T}^{\prime} \cup\{\alpha\} \not \models\left(\prod_{A^{\prime} \in \Sigma} A^{\prime} \sqcap\right.$ All $\left.\Sigma\right) \sqsubseteq E^{\prime}$ then for any $A \in \operatorname{sig}(\mathcal{T})$ and any $\Sigma$-concept $\exists r . G$ we have $\mathcal{T}^{\prime} \not \models \exists r . G \sqsubseteq \exists r$. $E^{\prime}$. So, this case is similar to the case of $E$ being pseudo-primitive considered above.

If $r \in \Sigma$ and $\mathcal{T}^{\prime} \cup\{\alpha\} \models\left(\prod_{A^{\prime} \in \Sigma} A^{\prime} \sqcap\right.$ All $\left.\Sigma\right) \sqsubseteq E^{\prime}$, it can be seen (using Theorem 6 and Lemma 1 ) that $\mathcal{T}^{\prime} \cup$ Noimply $_{\mathcal{T}, \Sigma} \models \xi_{A} \sqsubseteq \exists r . E^{\prime}$ for all $\xi_{A}$ except when $A$ has a definition $A \equiv \exists r . A^{\prime}$ in $\mathcal{T}$ and for all $\xi^{\prime} \in$ noimply $_{\mathcal{T}, \Sigma}\left(A^{\prime}\right)$ we have $\mathcal{T}^{\prime} \cup$ Noimply $_{\mathcal{T}, \Sigma} \not \models \xi^{\prime} \sqsubseteq E^{\prime}$ and $\operatorname{pre}_{\mathcal{T}^{\prime}}^{\Sigma}(E) \subseteq \operatorname{pre}_{\mathcal{T}}^{\Sigma}(\xi)$.

\section{E Computing uniform interpolants}

We state the result again.

Theorem 4. Let $\mathcal{T}$ be an acyclic terminology and $\Sigma$ a signature. Then there exists a uniform interpolant of $\mathcal{T}$ w.r.t. $\Sigma$. 
Proof. First we show that $\mathcal{T}_{\Sigma}=\mathcal{T}_{\Sigma}^{l} \cup \mathcal{T}_{\Sigma}^{r}$ is a uniform interpolant for $\mathcal{T}$ w.r.t. $\Sigma$ if $\operatorname{sig}\left(\mathcal{T}_{\Sigma}\right) \subseteq \Sigma$ and

(a) $\mathcal{T} \models C \sqsubseteq A$ if, and only if, $\mathcal{T}_{\Sigma}^{l}=C \sqsubseteq A$, for all $\Sigma$-concepts $C$ and $A \in \Sigma$;

(b) $\mathcal{T} \models A \sqsubseteq D$ if, and only if, $\mathcal{T}_{\Sigma}^{r} \models A \sqsubseteq D$, for all $\Sigma$-concepts $D$ and $A \in \Sigma$.

Suppose $\mathcal{T}_{\Sigma}$ has these properties. We show that for every $\Sigma$-implication $C \sqsubseteq D$ with $\mathcal{T} \models C \sqsubseteq D$ we obtain $\mathcal{T}_{\Sigma} \models C \sqsubseteq D$. The proof is by induction on $n=\operatorname{depth}(D)$. For $n=0, D$ is a conjunction of concept names, and so the claim is trivial because $\mathcal{T}_{\Sigma}=C \sqsubseteq A$ for all conjuncts $A$ of $D$. Consider $C \sqsubseteq D$ with $n+1=\operatorname{depth}(D)$. If $D$ is a conjunction of concepts, it is sufficient to show $\mathcal{T}_{\Sigma}=C \sqsubseteq D^{\prime}$ for each conjunct $D^{\prime}$ of $D$. Thus, it is sufficient to show the claim for $D=\exists r . D_{0}$. But if $\mathcal{T} \models C \sqsubseteq \exists r . D_{0}$, then, by Lemma 1 , there exists a conjunct $C^{\prime}$ of $C$ such that $C^{\prime}=A$, for a concept name $A$, and $\mathcal{T} \models A \sqsubseteq D$ or $C^{\prime}=\exists r . C_{0}$ and $\mathcal{T} \models C_{0} \sqsubseteq D_{0}$. In the first case we are done, in the second case the claim follows by induction.

In our construction of $\mathcal{T}_{\Sigma}$ we first consider (a). For each concept name $A \in$ $\operatorname{sig}(\mathcal{T})$ we construct a set $P_{\Sigma}(A)$ of $\Sigma$-concepts and general TBox $\mathcal{T}_{\Sigma, A}$ with $\operatorname{sig}\left(\mathcal{T}_{\Sigma, A}\right) \subseteq \Sigma$ such that

1. $\mathcal{T}=\mathcal{T}_{\Sigma, A}$, for all $A \in \operatorname{sig}(\mathcal{T})$

2. if $\mathcal{T} \models D \sqsubseteq A$ for a $\Sigma$-concept $D$ and $A \in \Sigma$, then $\mathcal{T}_{\Sigma, A} \models D \sqsubseteq A$.

3. $\mathcal{T}=D \sqsubseteq A$ for all $D \in P_{\Sigma}(A)$ and $A \in \operatorname{sig}(\mathcal{T})$;

4. if $\mathcal{T}=D \sqsubseteq A$ for a $\Sigma$-concept $D$ and $A \in \operatorname{sig}(\mathcal{T}) \backslash \Sigma$, then there exists $D^{\prime} \in P_{\Sigma}(A)$ such that $\mathcal{T}_{\Sigma, A} \models D \sqsubseteq D^{\prime}$.

The construction of $\mathcal{T}_{\Sigma, A}$ and $P_{\Sigma}(A)$ is shown in Figure 8. We show that they have the required properties. Points 1 and 3 are straightforward, so we concentrate on Points 2 and 4. Suppose $A$ is pseudo-primitive in $\mathcal{T}$ and $\mathcal{T} \models D \sqsubseteq A$.

- if $D$ is a concept name, Points 2 and 4 follow immediately from the definition.

- Suppose $D=D_{1} \sqcap D_{2}$. Then, by Lemma $1, \mathcal{T} \models D_{i} \sqsubseteq A$ for some $i \in\{1,2\}$. The claim follows by induction.

- Suppose $D=\exists r \cdot D^{\prime}$. Then, by Lemma $1, \mathcal{T} \not \models D \sqsubseteq A$, and the claim follows.

Suppose $A \equiv B_{1} \sqcap \cdots \sqcap B_{n} \in \mathcal{T}$ and $\mathcal{T} \models D \sqsubseteq A$. Assume first that $A \in \Sigma$. Then $\mathcal{T}=D \sqsubseteq B_{i}$ for all $i$. By induction,

- if $B_{i} \in \Sigma$, then $\mathcal{T}_{\Sigma, B_{i}} \models D \sqsubseteq B_{i}$;

- if $B_{i} \notin \Sigma$, then there exists $C_{i} \in P_{\Sigma}\left(B_{i}\right)$ such that $\mathcal{T}_{\Sigma, B_{i}} \models D \sqsubseteq C_{i}$.

Thus, from the definition of $\mathcal{T}_{\Sigma, A}$, we obtain

$$
\mathcal{T}_{\Sigma, A}=\prod_{B_{i} \notin \Sigma} C_{i} \sqcap \prod_{B_{i} \in \Sigma} B_{i} \sqsubseteq A
$$

and

$$
\mathcal{T}_{\Sigma, A} \models D \sqsubseteq \prod_{B_{i} \notin \Sigma} C_{i} \sqcap \prod_{B_{i} \in \Sigma} B_{i}
$$


Input: normalised acyclic terminology $\mathcal{T}$ and signature $\Sigma$.

For each $A \in \operatorname{sig}(\mathcal{T})$, construct sets $P_{\Sigma}(A)$ of $\Sigma$-concepts and general $\mathcal{E} \mathcal{L}$-TBox $\mathcal{T}_{\Sigma, A}$ inductively as follows:

- For $A$ pseudo-primitive in $\mathcal{T}$ :

- if $A \in \Sigma$, then $P_{\Sigma}(A)=\{A\}$ and $\mathcal{T}_{\Sigma, A}=\left\{B \sqsubseteq A \mid B \in \operatorname{pre}_{\mathcal{T}}^{\Sigma}(A)\right\}$

- if $A \notin \Sigma$, then $P_{\Sigma}(A)=\operatorname{pre}_{\mathcal{T}}^{\Sigma}(A)$ and $\mathcal{T}_{\Sigma, A}=\emptyset$.

- if $A$ is conjunctive in $\mathcal{T}$ and $A \equiv B_{1} \sqcap \cdots \sqcap B_{n} \in \mathcal{T}$ :

- if $A \in \Sigma$, then $P_{\Sigma}(A)=\{A\}$ and

$$
\mathcal{T}_{\Sigma, A}=\left\{C_{B_{1}} \sqcap \cdots \sqcap C_{B_{n}} \sqsubseteq A \mid C_{B_{i}} \in P_{\Sigma}\left(B_{i}\right), 1 \leq i \leq n\right\} \cup \bigcup_{1 \leq i \leq n} \mathcal{T}_{B_{i}, \Sigma} .
$$

- if $A \notin \Sigma$, then $P_{\Sigma}(A)=\left\{C_{B_{1}} \sqcap \cdots \sqcap C_{B_{n}} \mid C_{B_{i}} \in P_{\Sigma}\left(B_{i}\right), 1 \leq i \leq n\right\}$ and $\mathcal{T}_{\Sigma, A}=\bigcup_{1 \leq i \leq n} \mathcal{T}_{B_{i}, \Sigma}$.

- if $A \equiv \exists r . A^{\prime} \in \mathcal{T}$, then

- if $r \notin \Sigma$ and $A \notin \Sigma$, then $P_{\Sigma}(A)=\operatorname{pre}_{\mathcal{T}}^{\Sigma}(A), \mathcal{T}_{\Sigma, A}=\emptyset$.

- if $r \notin \Sigma$ and $A \in \Sigma$, then $P_{\Sigma}(A)=\{A\}, \mathcal{T}_{\Sigma, A}=\left\{B \sqsubseteq A \mid B \in \operatorname{pre}_{\mathcal{T}}^{\Sigma}(A)\right\}$;

- if $r \in \Sigma$ and $A \notin \Sigma$, then $P_{\Sigma}(A)=\operatorname{pre}_{\mathcal{T}}^{\Sigma}(A) \cup\left\{\exists r . E \mid E \in P_{\Sigma}\left(A^{\prime}\right)\right\}$, $\mathcal{T}_{\Sigma, A}=\mathcal{T}_{\Sigma, A^{\prime}}$.

- if $r \in \Sigma$ and $A \in \Sigma$, then $P_{\Sigma}(A)=\{A\}$,

$$
\mathcal{T}_{\Sigma, A}=\left\{B \sqsubseteq A \mid B \in \operatorname{pre}_{\mathcal{T}}^{\Sigma}(A)\right\} \cup \mathcal{T}_{\Sigma, A^{\prime}} \cup\left\{\exists r . E \sqsubseteq A \mid E \in P_{\Sigma}\left(A^{\prime}\right)\right\}
$$

Fig. 8. Computing uniform interpolant for implications $C \sqsubseteq A$

Hence $\mathcal{T}_{\Sigma, A}=D \sqsubseteq A$. The case $A \notin \Sigma$ is similar and left to the reader.

Suppose $A \equiv \exists r . A^{\prime} \in \mathcal{T}$ and $\mathcal{T} \models D \sqsubseteq A$. Assume first that $A \in \Sigma$. By Lemma 1, there are two cases:

(1) there exists an atomic conjunct $B$ of $D$ such that $\mathcal{T} \models B \sqsubseteq A$. Then $B \sqsubseteq A \in \mathcal{T}_{\Sigma, A}$ and, therefore, $\mathcal{T}_{\Sigma, A}=D \sqsubseteq A$.

(2) there exists a conjunct $\exists r . D^{\prime}$ of $D$ such that $\mathcal{T} \equiv D^{\prime} \sqsubseteq A^{\prime}$. Then $r \in \Sigma$. Assume first $A^{\prime} \in \Sigma$. Then $\mathcal{T}_{\Sigma, A^{\prime}} \models D^{\prime} \sqsubseteq A^{\prime}$. Hence $\mathcal{T}_{\Sigma, A}=D^{\prime} \sqsubseteq A^{\prime}$ and we obtain $\mathcal{T}_{\Sigma, A}=D \sqsubseteq A$. Now assume $A^{\prime} \notin \Sigma$. We find $C \in P_{\Sigma}\left(A^{\prime}\right)$ such that $\mathcal{T}_{\Sigma, A^{\prime}} \models D^{\prime} \sqsubseteq C$. We have $\exists r . C \sqsubseteq A \in \mathcal{T}_{\Sigma, A}$. But then $\mathcal{T}_{\Sigma, A} \models \exists r . D^{\prime} \sqsubseteq A$. The case $A \notin \Sigma$ is similar and left to the reader.

We now consider (b). For each concept name $A \in \operatorname{sig}(\mathcal{T})$ we construct a set $Q_{\Sigma}(A)$ of $\Sigma$-concepts and general $\Sigma$-TBox $\mathcal{T}_{\Sigma, A}^{r}$ such that

1. $\mathcal{T}=\mathcal{T}_{\Sigma, A}^{r}$ for all $A \in \operatorname{sig}(\mathcal{T})$;

2. if $\mathcal{T}=A \sqsubseteq D$ for a $\Sigma$-concept $D$ and $A \in \Sigma$, then $\mathcal{T}_{\Sigma, A}^{r}=A \sqsubseteq D$;

3. $\mathcal{T}=A \sqsubseteq D$ for all $D \in Q_{\Sigma}(A)$ and $A \in \operatorname{sig}(\mathcal{T})$;

4. if $\mathcal{T} \models A \sqsubseteq D$ for a $\Sigma$-concept $D$ and $A \in \operatorname{sig}(\mathcal{T}) \backslash \Sigma$, then there exists a conjunction $D$ of concepts in $Q_{\Sigma}(A)$ such that $\mathcal{T}_{\Sigma, A}^{r} \models D^{\prime} \sqsubseteq D$.

In this case we assume, w.l.o.g., that $\mathcal{T}$ is a strongly normalised acyclic terminology: An acyclic terminology is strongly normalised if its axioms are of the form 
Input: strongly normalised acyclic terminology $\mathcal{T}$ and signature $\Sigma$.

For each $A \in \operatorname{sig}(\mathcal{T})$, construct sets $Q_{\Sigma}(A)$ of $\Sigma$-concepts and general TBox $\mathcal{T}_{\Sigma, A}^{r}$ inductively as follows:

- For $A$ is primitive in $\mathcal{T}$ :

- if $A \in \Sigma$, then $Q_{\Sigma, A}=\{A\}$ and $\mathcal{T}_{\Sigma, A}^{r}=\left\{A \sqsubseteq B \mid A \in \operatorname{pre}_{\mathcal{T}}^{\Sigma}(B)\right\}$.

- if $A \notin \Sigma$, then $Q_{\Sigma, A}=\left\{B \in \Sigma \mid A \in \operatorname{pre}_{\mathcal{T}}^{\Sigma}(B)\right\}$ and $\mathcal{T}_{\Sigma, A}=\emptyset$.

- if $A$ is conjunctive in $\mathcal{T}$ and $A \equiv B_{1} \sqcap \cdots \sqcap B_{n} \in \mathcal{T}$, then

- if $A \in \Sigma$, then $Q_{\Sigma}(A)=\{A\}$ and

$$
\mathcal{T}_{\Sigma, A}^{r}=\left\{A \sqsubseteq D \mid D \in \bigcup_{1 \leq i \leq n} Q_{\Sigma}\left(B_{i}\right)\right\} \cup\left\{A \sqsubseteq B \mid A \in \operatorname{pre}_{\mathcal{T}}^{\Sigma}(B)\right\} \cup \bigcup_{1 \leq i \leq n} \mathcal{T}_{B_{i}, \Sigma}^{r}
$$

- if $A \notin \Sigma$, then

$$
\begin{gathered}
Q_{\Sigma}(A)=\bigcup_{1 \leq i \leq n} Q_{\Sigma}\left(B_{i}\right) \cup\left\{B \mid A \in \operatorname{pre}_{\mathcal{T}}^{\Sigma}(B)\right\} \\
\mathcal{T}_{\Sigma, A}^{r}=\bigcup_{1 \leq i \leq n} \mathcal{T}_{B_{i}, \Sigma}^{r}
\end{gathered}
$$

- if $A \equiv \exists r \cdot A^{\prime} \in \mathcal{T}$, then

- if $r \notin \Sigma$ and $A \notin \Sigma$, then $Q_{\Sigma}(A)=\left\{B \mid A \in \operatorname{pre}_{\mathcal{T}}^{\Sigma}(B)\right\}$ and $\mathcal{T}_{\Sigma, A}^{r}=\emptyset$.

- if $r \notin \Sigma$ and $A \in \Sigma$, then $Q_{\Sigma}(A)=\{A\}, \mathcal{T}_{\Sigma, A}^{r}=\left\{A \sqsubseteq B \mid A \in \operatorname{pre}_{\mathcal{T}}(B)\right\}$;

- if $r \in \Sigma$ and $A \notin \Sigma$, then $Q_{\Sigma}(A)=\left\{B \mid A \in \operatorname{pre}_{\mathcal{T}}^{\Sigma}(B)\right\} \cup\{\exists r . E \mid E \in$ $\left.Q_{\Sigma}\left(A^{\prime}\right)\right\}, \mathcal{T}_{\Sigma, A}=\mathcal{T}_{\Sigma, A^{\prime}}$.

- if $r \in \Sigma$ and $A \in \Sigma$, then $Q_{\Sigma}(A)=\{A\}$,

$$
\mathcal{T}_{\Sigma, A}^{r}=\left\{A \sqsubseteq B \mid A \in \operatorname{pre}_{\mathcal{T}}(B)\right\} \cup \mathcal{T}_{\Sigma, A^{\prime}}^{r} \cup\left\{A \sqsubseteq \exists r . E \mid E \in Q_{\Sigma}\left(A^{\prime}\right)\right\}
$$

Fig. 9. Computing uniform interpolant for $A \sqsubseteq C$

$A \equiv \exists r . B$, where $B$ is a concept name or of the form $A \equiv B_{1} \sqcap B_{2}$, where $B_{1}$ and $B_{2}$ are concept names. Lemma 3 is easily modified so as to cover strongly normalised acyclic terminology. (For example, axioms of the form $A \sqsubseteq C$ are replaced by axioms $A \equiv X \sqcap C$, where $X$ is a fresh concept name). The construction of $Q_{\Sigma}(A)$ and $\mathcal{T}_{\Sigma, A}^{r}$ is shown in Figure 9.

We show that they have the required properties. Points 1 and 3 are straightforward, so we concentrate on Points 2 and 4 . Suppose $A$ is primitive in $\mathcal{T}$ and $\mathcal{T} \models A \sqsubseteq D$.

- if $D$ is a concept name, Points 2 and 4 follow immediately from the definition.

- Suppose $D=D_{1} \sqcap D_{2}$. Then the claim follows inductively from $\mathcal{T} \models A \sqsubseteq D_{i}$ for $i=1,2$.

- Suppose $D=\exists r . D^{\prime}$. Then $\mathcal{T} \not \models A \sqsubseteq D$ and the claim follows.

Suppose $A \equiv B_{1} \sqcap B_{2} \in \mathcal{T}$ and $\mathcal{T} \models A \sqsubseteq D$.

- the inductive steps for $D$ a concept name or $D=D_{1} \sqcap D_{2}$ are again straightforward and left to the reader. 
- Suppose $D=\exists r . D^{\prime}$. Then, by Lemma 1 , there exists $B_{i}$ such that $\mathcal{T} \models B_{i} \sqsubseteq$ $\exists r . D^{\prime}$. The claim follows by induction.

Suppose $A \equiv \exists r . A^{\prime} \in \mathcal{T}$ and $\mathcal{T}=A \sqsubseteq D$.

- the inductive steps for $D$ a concept name or $D=D_{1} \sqcap D_{2}$ are again straightforward and left to the reader.

- Suppose $D=\exists s . D^{\prime}$. Then, by Lemma $1, r=s$ and $\mathcal{T} \models A^{\prime} \sqsubseteq D^{\prime}$. Again the claim follows by induction.

Finally, it is now easy to show that $\mathcal{T}_{\Sigma}^{l}=\bigcup_{A \in \Sigma} \mathcal{T}_{\Sigma, A}$ and $\mathcal{T}_{\Sigma}^{r}=\bigcup_{A \in \Sigma} \mathcal{T}_{\Sigma, A}^{r}$ are as required. 